\title{
THE BOTTOM FAUNA OF GREAT WEST BAY
}

\author{
By N. A. Holme, B.A. \\ Zoologist at the Plymouth Laboratory
}

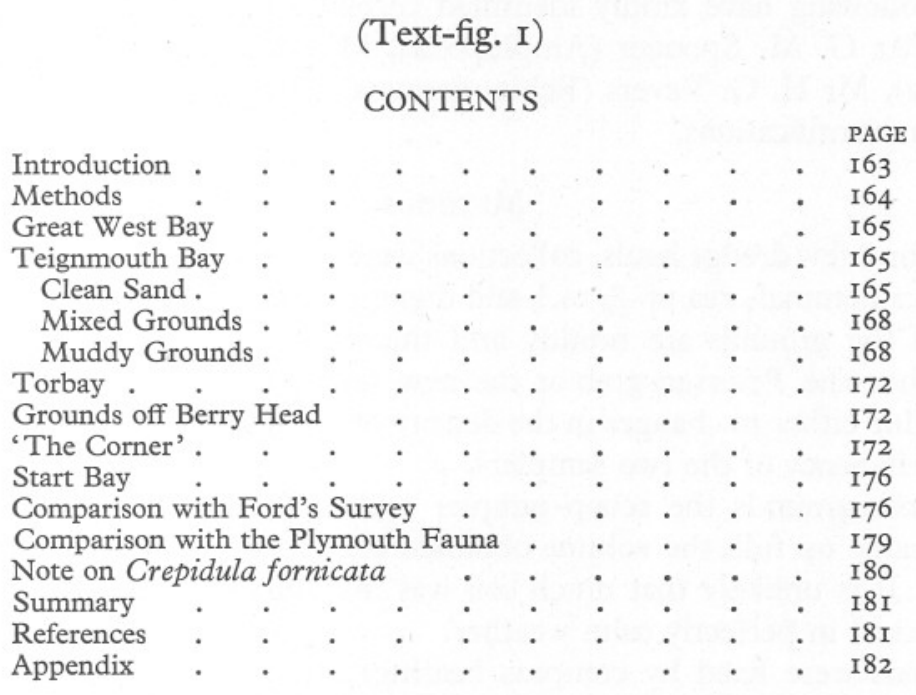

\section{INTRODUCTION}

At the end of the last century extensive investigations were made of the trawling grounds in the bay between Start Point and Portland, sometimes known as Great West Bay (see Stead, I896; Holt, I898; Garstang, I903; Kyle, I903; and Todd, I903). Although these deal mainly with the food-fish of the area, Todd gives an account of invertebrates brought up in the trawl. Since the trawl mainly samples the 'epifauna' of the sea-bottom, no comparison will be made of Todd's results with those obtained in the grab surveys to be described.

In November 1923, Mr E. Ford made a survey of the bottom fauna of the Bay, using a $\frac{1}{10} \mathrm{~m} .{ }^{2}$ Petersen grab. With the exception of a number of stations in Start Bay (see Ford, 1925), the results were not published. Mr Ford has kindly lent me the notes on his collections, some of which are included in this paper.

In the summer of 1948 a cruise was made in the western side of the Bay, samples being taken at thirty-seven stations with the bottom-sampler described by Holme (1949). At each station between one and five hauls were made, each covering an area of $\frac{1}{20} \mathrm{~m} .{ }^{2}$. The results are in the main very similar to those of Ford, showing that it is possible to make a reasonably reliable qualitative survey of a fairly large area using these methods. They also show that the bottom-fauna has not changed to any extent in the past 25 years.

The survey was made during the term of a D.S.I.R. research grant at the 
Plymouth Laboratory. I am grateful to the Director and staff for facilities afforded during this period.

I am indebted to Captain C. A. Hoodless and the crew of R.V. Sabella for their assistance in taking the samples, and to Miss U. M. Grigg for help in sorting and preserving specimens during the cruise.

The following have kindly identified certain species: Miss S. M. Davies (Eone), Mr G. M. Spooner (Amphipoda), Miss U. M. Grigg (some of the Mollusca), Mr H. G. Vevers (Echinodermata). The author is responsible for the other identifications.

\section{METHODS}

Except for a few dredge hauls, collections were made with the 'scoop-sampler', covering a nominal area of $\frac{1}{20} \mathrm{~m} .{ }^{2}$, and digging to a maximum depth of $15 \mathrm{~cm}$. Many of the grounds are muddy and therefore fairly suitable for working with either the Petersen grab or the new sampler. Quantitative differences may be due either to changes in the density of the fauna over 25 years or to the relative efficiency of the two samplers.

On most grounds the scoop-sampler took a fairly adequate sample, but seldom came up full, the volume obtained corresponding to a 'bite' of depth $6-8 \mathrm{~cm}$. It is unlikely that much soil was lost during hauling as all stations were worked in perfectly calm weather.

Positions were fixed by compass bearings on to the shore, which was in sight at all but one station. Station lists are given in Tables XI and XII (pp. I82-3). The writer's soundings have been reduced to chart datum.

Since tidal streams are weak the hauls at each station could be taken without the ship drifting more than perhaps $100 \mathrm{~m}$., usually much less.

Collections at each station were put together and sieved through a mesh of $\mathrm{I}, 2$, or $3 \mathrm{~mm}$., depending on the grade of soil. Smaller Crustacea and worms may have been lost when the larger-meshed sieves were employed. A soil sample from each station has been kept.

At certain stations, where the bulk of the sievings was great, only a proportion was kept for examination. To this was added any large or conspicuous animals seen in the rest of the sample. The numbers recorded at these stations are, therefore, not strictly quantitative, and can only be taken to represent the minimum numbers present in the sample. Owing to rather hasty preservation, inevitable when working through large quantities of material at sea, some of the smaller animals have not been identified further than the genus. Other specimens may, however, have been sufficiently well preserved to enable specific identification. Thus the recording of Pectinaria koreni and Pectinaria sp. from one station does not necessarilymean that more than one species was present.

Nomenclature usually follows the Plymouth Marine Fauna (Mar. Biol. Assoc., 193I). 


\section{GREAT WeST BAY}

Great West Bay is 48 miles across from Start Point to Portland, and extends 20 miles inwards. Its area is about 650 square miles. The western side is sheltered from westerly winds by the coastline; this and the absence of Atlantic swell make the sea rather less rough than off Plymouth.

Tidal currents in the Bay are weaker than in the main English Channel, but reach over 2 knots $(3.7 \mathrm{~km}$. $/ \mathrm{hr}$.) at Start Point and Portland Bill. Off Berry Head the current reaches only $\frac{3}{4}$ knot, while in Teignmouth Bay it is scarcely felt.

The bottom is variable, but coarse deposits are rare and localized. The most significant feature is the large amount of mud deposited, particularly off Berry Head and in Torbay. In parts of Start Bay and on 'the Corner', however, the soil is fairly clean, consisting of coarse sand or fine gravel.

The rivers Exe, Teign and Dart empty into the Bay, but do not affect the salinity outside their estuaries.

The area investigated will be considered under five headings: Teignmouth Bay, Torbay (worked only by Mr Ford), grounds off Berry Head, 'the Corner' fishing grounds, and Start Bay.

\section{TeIgnMouth BAY}

Teignmouth Bay extends from the Orestone to Straight Point, and has a maximum depth of c. $22 \mathrm{~m}$. Over much of the bay the depth is about $20 \mathrm{~m}$. The bay is exposed to the south and east, but sheltered from the north and west by the coastline.

Three fairly distinct types of bottom are found: clean sand; mixed grounds of sand, mud and gravel; and mud.

\section{Clean Sand}

This occurs in a belt parallel to the shore off Dawlish and Exmouth. Farther offshore the sand gives way to gravel deposits. The depth is less than II $\mathrm{m}$. Towards Dawlish the sand is finer and slightly muddy.

In 1948 the following stations were worked in this area: I, 2, I2 and I4-I6 (Table I). The fauna off Exmouth (Stations I and 2) is poor, but becomes richer towards Dawlish.

Species characteristic of the area are: Sigalion mathildae, Nephthys sp., Magelona papillicornis, Iphinoë trispinosa, Bathyporeia spp., Tellina fabula, Donax vittatus, ? Spisula subtruncata and Ensis sp. ${ }^{1}$ Echinocardium cordatum is quite common at Station I6, but is rare or absent elsewhere.

Ford did not work this area.

${ }^{1}$ A note on the species of Ensis to be found in the area will be published shortly. 


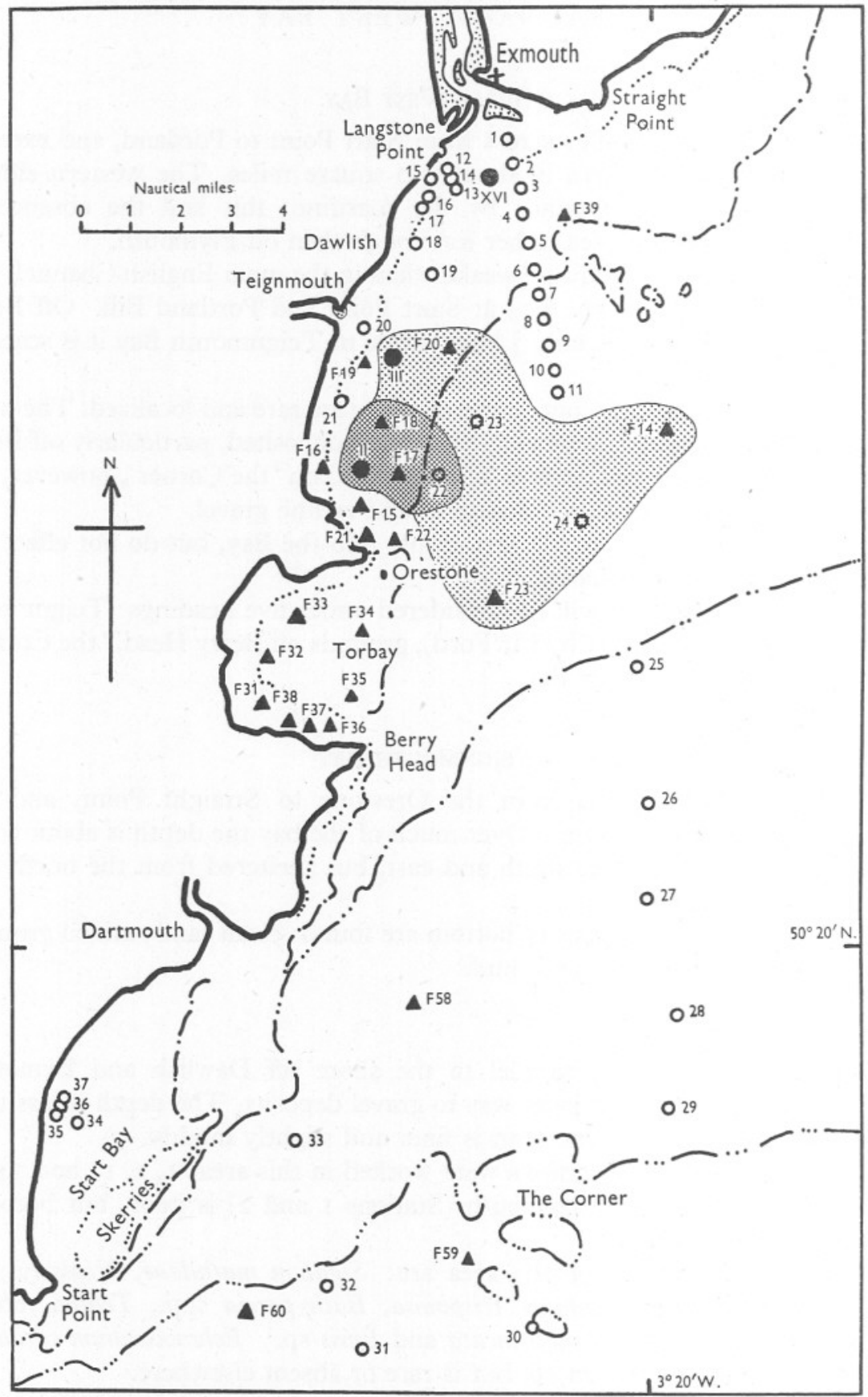

Fig. I. Map of the western side of Great West Bay, showing stations worked by Mr Ford in I923 and by the author in I948. A, I923 stations (Petersen grab); O, I948 stations (scoop-sampler); $\bullet$, I948 dredge-hauls. ..., 5-fathom line; ---, Io-fathom line; $-\cdots-, 20$-fathom line; _...- 30-fathom line. $\%$, area occupied by living Turritella; : area where dead Turritella shells are abundant. 


\section{Table I. Fauna of Fine Clean Sand in Teignmouth Bay}

\section{Notes on Stations}

Station I. 29. vii. 48. Exmouth Church, $349^{\circ}, \mathrm{r} \cdot 2^{\prime}$. Clean sand.

Station 2. 29. vii. 48. Exmouth Church, $349^{\circ}, I^{\prime} 7^{\prime}$. Gravel and sand.

Station I2. 30. vii. 48 . Langstone Point, $334^{\circ}, 0 \cdot 35^{\prime}$. Clean sand.

Station I4. 30. vii. 48. Langstone Point, $334^{\circ}, 0.55^{\prime}$. Clean fine sand overlying gravel.

Station I5. 30. vii. 48. Langstone Point, $022^{\circ}, 0.55^{\prime}$. Clean fine sand and some gravel. Donax picked out, and fauna sorted from two-thirds of total gravel.

Station I6. 30. vii. 48 . Langstone Point, $013^{\circ}, 0.9^{\prime}$. Sand.

The nominal area of each haul is $0.05 \mathrm{~m}$.

\begin{tabular}{|c|c|c|c|c|c|c|c|}
\hline Station & . & I & 2 & I2 & 14 & I5 & I6 \\
\hline Depth (m.) & .. & $5 \cdot 5$ & 10.5 & $5 \cdot 5$ & $7 \cdot 5$ & 5 & 10.5 \\
\hline Area $\left(\mathrm{m} .^{2}\right)$ & . & 0.15 & 0.05 & 0.1 & 0.05 & 0.05 & 0.2 \\
\hline Sieve (mm.) & . & I & 3 & I & I & I & I \\
\hline \multicolumn{8}{|c|}{ COELENTERATA } \\
\hline $\begin{array}{l}\text { ?Peachia } \\
\text { ?Sagartia }\end{array}$ & & . & $\cdots$ & . & .. & . & $3 \mathrm{j}$. \\
\hline ?Sagartia & & $\cdots$ & $\cdots$ & . & . & $\cdots$ & I \\
\hline \multicolumn{8}{|l|}{ NEMERTINI } \\
\hline $\begin{array}{l}\text { Nemertin } \\
\text { PoLyCHAETA }\end{array}$ & & $\cdots$ & $\cdots$ & I & $\cdots$ & $\cdots$ & $\cdots$ \\
\hline \multicolumn{8}{|c|}{ Polychaeta } \\
\hline Sigalion $n$ & dae & $\cdots$ & $\cdots$ & 2 & $\cdots$ & $\cdots$ & c. 4 \\
\hline Sthenelais & & $\ddot{r}$ & $\cdots$ & $\cdots$ & $\cdots$ & $\cdots$ & I \\
\hline Phyllodoce & & $\begin{array}{l}\mathrm{I} \\
.\end{array}$ & 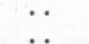 & $\because$ & $\cdots$ & $\cdots$ & 3 \\
\hline Nereis sp. & & c. $\ddot{1} \dot{3}$ & $\ddot{\cdots}$ & c. $\dot{3}$ & $\cdots$ & $\cdots$ & I \\
\hline Nephthys & & . 13 & $\cdots$ & $\begin{array}{r}\text { c. } 3 \\
\ldots\end{array}$ & $\cdots$ & $\cdots$ & 4 \\
\hline Scoloplos & & $\cdots$ & $\cdots$ & $\begin{array}{l}\cdots \\
\cdots\end{array}$ & $\cdots$ & $\cdots$ & I \\
\hline Nerine sp & licornis & c. 40 & . & $\ddot{6} 4$ & $\ddot{s}$ & $\ddot{i}$ & I \\
\hline $\begin{array}{l}\text { Magelona } \\
\text { ?wenia tu }\end{array}$ & & . & . & $\begin{array}{l}04 \\
\cdots\end{array}$ & 5 & IO & IO \\
\hline $\begin{array}{l}\text { ?Owenia tu } \\
\text { Pectinaria }\end{array}$ & & ?I & 2 & $\cdots$ & $\ddot{\cdots}$ & $\ddot{x}$ & 3 \\
\hline ?Lanice tul & & . & I. & . & . & 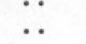 & $\ddot{6}$ \\
\hline Terebellic & & . & Ij. & . & . & . & . \\
\hline Polychaet & & I & I & 3 & $\cdots$ & I & I \\
\hline \multicolumn{8}{|l|}{ CRUStacea } \\
\hline Iphinoë tr & & 20 & I & 3 & I & $\cdots$ & $\cdots$ \\
\hline Diastylis & & 2 & $\cdots$ & $\because$ & $\cdots$ & $\cdots$ & I \\
\hline Diastylis & & $\because$ & $\cdots$ & I & $\cdots$ & $\cdots$ & $\cdots$ \\
\hline Pseudocun & caria & I & $\cdots$ & $\cdots$ & $\cdots$ & $\cdots$ & $\ddot{r}$ \\
\hline Ampelisca & $\begin{array}{l}\text { cornis } \\
\text { illiamsoniana }\end{array}$ & $\ddot{4}$ & $\cdots$ & $\ddot{z}$ & $\because$ & $\ddot{I}$ & 3 \\
\hline Bathypore & $\begin{array}{l}\text { illiamsoniana } \\
\text { gans }\end{array}$ & $\begin{array}{l}4 \\
\ldots\end{array}$ & $\because$. & $\begin{array}{l}3 \\
3\end{array}$ & $\cdots$ & $? 3$ & $\ddot{I}$ \\
\hline $\begin{array}{l}\text { Bathypore } \\
\text { Urothoë g }\end{array}$ & $\begin{array}{l}\text { gans } \\
\text { dii }\end{array}$ & $\ddot{m}$ & $\cdots$ & $\begin{array}{l}3 \\
3\end{array}$ & $\because$ & 3 & $\ldots$ \\
\hline Leucothoë & orgi & . & . & $\ldots$ & . & . & $\ddot{4}$ \\
\hline Nototropi & nmerdami & I & .. & . & $\cdots$ & . & . \\
\hline Gammaru & sta & I & $\cdots$ & . & $\cdots$ & $\cdots$ & . \\
\hline Amphipo & det. & $\cdots$ & $\because$ & 5 & $\cdots$ & $\cdots$ & . \\
\hline Prawn & & $\cdots$ & I & $\cdots$ & $\cdots$ & $\cdots$ & $\because$ \\
\hline Corystes c & launus & $\cdots$ & $\cdots$ & $\cdots$ & $\cdots$ & $\cdots$ & $\mathbf{r j}$. \\
\hline \multicolumn{8}{|l|}{ MOLLUSCA } \\
\hline Nucula ni & & $\because$ & $\cdots$ & $\cdots$ & $\cdots$ & . & I \\
\hline PPecten mo & & Ij. & $\cdots$ & $\cdots$ & $\cdots$ & . & . \\
\hline Chlamys & laris & Ij. & $\cdots$ & $\cdots$ & $\cdots$ & $\cdots$ & $\cdots$ \\
\hline Montacut & uginosa & $\ddot{0}$ & $\cdots$ & $\because$ & $\cdots$ & $\cdots$ & 2 \\
\hline Tellina $\mathrm{fa}$ & & 2). & $\cdots$ & I). & $\cdots$ & $\cdots$ & $2 j$. \\
\hline Abra alba & & $\ddot{4 i}$ & $\because$ & $\because$ & $\because$ & $\cdots$ & I \\
\hline Abra sp. & & $\begin{array}{l}4) . \\
\cdots\end{array}$ & 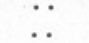 & $\ddot{2 j}$. & $\begin{array}{l}\cdots \\
\cdots\end{array}$ & $\ddot{I}$ & $\ddot{x}$ \\
\hline $\begin{array}{l}\text { Donax vit } \\
\text { ?Spisula st }\end{array}$ & cata & $6 \mathrm{j}$. & . & $\cdots$ & . & . & $\ddot{\mathrm{ij}}$. \\
\hline Lutraria & phons) & . & . & . & . & . & 2 \\
\hline Mysia un & & $\cdots$ & .. & . & . & $\cdots$ & I \\
\hline Chione sti & & $\cdots$ & $\cdots$ & $\cdots$ & $\cdots$ & $\cdots$ & I \\
\hline ?Cardium & tum & Ij. & $\cdots$ & $\cdots$ & $\cdots$ & $\cdots$ & . \\
\hline ?Cardium & ulatum & Ij. & $\cdots$ & $\cdots$ & $\cdots$ & $\cdots$ & . \\
\hline Ensis sp. & & $4 j$. & $\cdots$ & 2. & . & $\cdots$ & 2 \\
\hline Lamellib & s indet. & 8 & $\cdots$ & $4 j$. & $\cdots$ & . & I \\
\hline Philine af & & $\cdots$ & $\therefore$ & I & $\cdots$ & $\cdots$ & $I$ \\
\hline \multicolumn{8}{|l|}{ POLYZOA. } \\
\hline Cellaria s & & + & $\cdots$ & $\cdots$ & $\cdots$ & $\cdots$ & $\cdots$ \\
\hline \multicolumn{8}{|c|}{ ECHINODERMATA } \\
\hline Acrocnido & hiata & $\cdots$ & . & $\cdots$ & .. & $\cdots$ & 2 \\
\hline Echinocar & cordatum & . & . & $\mathrm{Ij}$. & $\cdots$ & $\cdots$ & 3 \\
\hline Labidopla & itata & $\cdots$ & $\cdots$ & $\cdots$ & $\cdots$ & $\cdots$ & 5 \\
\hline
\end{tabular}

?, identity doubtful; j., small specimens; +, present, but numbers uncertain; + +, abundant. 


\section{Mixed Grounds}

These occur off-shore in Teignmouth Bay in a depth of 10-23 m. Stations $3^{-I I}, \mathrm{I} 3$ and 23 were worked in this area (Tables II and III). Deposits are of sand, fine shell fragments, and mud, sometimes mixed with fine gravel.

Characteristic species are: Cerianthus lloydi, Melinna palmata, Phascolion strombi (often in empty Turritella shells), Eupagurus spp., Aloidis gibba, Cultellus pellucidus and Amphiura filiformis.

Egg-clusters of Turritella communis were common at this time, and when trawling the meshes of the net came up covered with them. The presence of the Salcombe commensal, Lepidasthenia argus, at Station 3 is of interest. Although not taken in the grab hauls, Gibbula magus was found to be abundant off Langstone Point (dredge-haul XVI). A number of dredgings in the area showed that it was common only at this one station, where the bottom was of gravel.

Ford's Station 39, in this area, was evidently near a rocky ledge running southward from Straight Point. This is indicated by the presence of Psammechinus miliaris in the sample.

\section{Muddy Grounds}

These occur between Dawlish and the Orestone. Between Dawlish and Teignmouth the ground becomes progressively muddier, and north of the Orestone there is a deposit of mud in which Turritella communis is very abundant. The depth is between IO and $22 \mathrm{~m}$., and Stations I7-22 were worked in this area (Table IV).

Where living Turritella is abundant, only two or three other species are found. These include Eupagarus bernhardus which inhabits the empty shells. The estimated extent of the Turritella bed is shown in Fig. I. At some stations living Turritella were abundant, at others large numbers of dead and worn shells were taken. In dredge-haul II living shells formed such a large proportion of the catch that they half-filled a galvanized iron bath. In dredge-haul III many white and dead shells were taken, but very few living ones occurred.

$\mathrm{Mr}$ Ford worked a number of stations in this bed, and the area shown on Fig. I is based on collections made on both cruises. There seems to be an area north of the Orestone where the animals live in abundance, from which dead shells are swept to the north and east by waves or currents.

It is of interest to note that Kyle (1903) records oysters (Ostrea edulis) from this area:

\footnotetext{
'From Hope's Nose to off Teignmouth there is a stretch of hard ground on which oysters are fairly abundant. As showing the trend of the currents in Teignmouth Bay, it may be mentioned that the empty shells congregate in masses behind the Orestone on the Torbay side.' (Hope's Nose is the headland opposite the Orestone).
}

Oyster shells are now rare or absent in Teignmouth Bay. 


\section{Table II. Fauna of Mixed Muddy and Gravel Grounds in Teignmouth Bay}

Note. With most samples large animals were picked out, and a fraction of the sievings sorted (see p. I64). Numbers are those picked out, together with the subsample, which has not been multiplied by a factor.

Station 3. 29. vii. 48. Exmouth Church, $348^{\circ} ; 2 \cdot 2^{\prime}$. Muddy sand and pebbles, overlying grey clay. One-quarter sorted.

Station 4. 29. vii. 48. Exmouth Church, $349^{\circ}, 2 \cdot 7^{\prime}$. Mud and a few pebbles. One-half sorted.

Station 5. 29. vii. 48. Exmouth Church, $349^{\circ}, 3 \cdot 3^{\prime}$. Fine shell fragments and sand. All sorted.

Station 6. 29. vii. 48. Exmouth Church, $349^{\circ}, 3 \cdot 8^{\prime}$. Muddy sand, gravel, and few shell fragments. Three-quarters sorted.

Station 7. 29. vii. 48. Exmouth Church, $349^{\circ}, 4 \cdot 3^{\prime}$. Fairly fine muddy sand. Small piece of red sandstone. One-quarter sorted.

Station 8. 29. vii. 48. Exmouth Church, $349^{\circ}, 4 \cdot 8^{\prime}$. Fairly fine muddy sand, with sandstone fragments. All sorted.

\begin{tabular}{|c|c|c|c|c|c|c|c|}
\hline Station & . & 3 & 4 & 5 & 6 & 7 & 8 \\
\hline Depth (m.) & . & I4 & $17 \cdot 5$ & 19 & I9 & $20 \cdot 5$ & $2 I \cdot 5$ \\
\hline Area $\left(\mathrm{m} .{ }^{2}\right)$ & . & 0.15 & $O \cdot I$ & $O \cdot I$ & 0.15 & $O \cdot I$ & 0.15 \\
\hline Sieve (mm.) & $\cdots$ & 3 & 3 & 3 & 3 & 3 & 3 \\
\hline \multicolumn{8}{|c|}{ COELENTERATA } \\
\hline Hydroid & & .. & $\ddot{\theta}$ & $\cdots$ & + & . & $\because$ \\
\hline Cerianthus & $y d i$ & I & 2 & $\cdots$ & $\cdots$ & 4 & 2 \\
\hline ?Peachia ha & & Ij. & $\cdots$ & $\cdots$ & $\cdots$ & $\cdots$ & $\cdots$ \\
\hline \multicolumn{8}{|l|}{ PORIFERA } \\
\hline Sponge & & $\cdots$ & $\cdots$ & $\cdots$ & $\cdots$ & $\cdots$ & I \\
\hline \multicolumn{8}{|l|}{ POLYCHAETA } \\
\hline Lepidasthe & argus & I & $\cdots$ & $\cdots$ & $\cdots$ & $\cdots$ & $\cdots$ \\
\hline Sthenelais & & I & $\cdots$ & $\cdots$ & $\ddot{3}$ & $\cdots$ & $\ddot{2}$ \\
\hline ?Glycera co & oluta & $\cdots$ & $\ddot{v}$ & $\cdots$ & 3 & $\cdots$ & 2 \\
\hline Glycera sp & unicornis & $\ddot{r}$ & I & $\ddot{r}$ & $\ddot{T}$ & $\cdots$ & $\cdots$ \\
\hline Nematoner & $\begin{array}{l}\text { unicornis } \\
\text { is sp. }\end{array}$ & I & . & I & I & $\cdots$ & $\because$ \\
\hline Lumbricon & $\begin{array}{l}\text { is } \mathrm{sp} \text {. } \\
\text { us } \mathrm{sp} \text {. }\end{array}$ & $\cdots$ & $\cdots$ & $\cdots$ & I & $\cdots$ & I \\
\hline Staurocepl & us sp. & $\ddot{v}$ & $\cdots$ & $\cdots$ & I & $\cdots$ & $\cdots$ \\
\hline $\begin{array}{l}\text { Stylaroide } \\
\text { Scalibregm }\end{array}$ & nflatum & $\begin{array}{l}1 \\
\ldots\end{array}$ & $\begin{array}{l}\cdots \\
\ldots\end{array}$ & $\ddot{I}$ & $\cdots$ & $\because$ & $\cdots$ \\
\hline & & $\because$ & $\ddot{I}$ & $\begin{array}{l}1 \\
\mathrm{I}\end{array}$ & $\cdots$ & $\cdots$ & $\cdots$ \\
\hline $\begin{array}{l}\text { ?Owenia tu } \\
\text { Pectinaria }\end{array}$ & reni & $\ldots$ & $\ldots$ & $\ldots$ & $\because$ & $\because$ & $\ddot{r}$ \\
\hline $\begin{array}{l}\text { Pectinaria } \\
\text { Pectinaria }\end{array}$ & ricoma & . & . & I & $\ldots$ & $\cdots$ & I \\
\hline $\begin{array}{l}\text { Pectinaria } \\
\text { Melinna }\end{array}$ & nata & 2 & I6 & 4 & 6 & 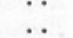 & $\ddot{I}$ \\
\hline $\begin{array}{l}\text { Melinna p } \\
\text { Terebellic }\end{array}$ & & $\ldots$ & .. & Ij. & .. & $\cdots$ & \\
\hline & & .. & 5 & I & 2 & $\because$ & $\because$ \\
\hline \multirow{2}{*}{\multicolumn{8}{|c|}{ GEPHYREA }} \\
\hline & & & & & & & \\
\hline Phascoloso & $\begin{array}{l}\text { elongatum } \\
\text { vulgare }\end{array}$ & $\ldots$ & $\ddot{p}$ & $\cdots$ & $\cdots$ & $\cdots$ & $\cdots$ \\
\hline Phascoloso & $\begin{array}{l}\text { vulgare } \\
\text { rombi }\end{array}$ & $\cdots$ & 3 & $\ddot{x}$ & $\ddot{v}$ & $\cdots$ & $\cdots$ \\
\hline \multirow{2}{*}{\multicolumn{8}{|c|}{ CRUSTACEA }} \\
\hline & & & & & & & \\
\hline Amphipoc & $\begin{array}{l}\text { indet. } \\
\text { anensis }\end{array}$ & $\because$ & . & I & I & 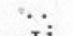 & $\cdots$ \\
\hline Eupagurus & $\begin{array}{l}\text { anensis } \\
\text { mboides }\end{array}$ & $\begin{array}{l}\cdots \\
\ldots\end{array}$ & $\cdots$ & $\ddot{\mathrm{i}}$ & $\cdots$ & I). & $\cdots$ \\
\hline Gonoplax & mbordes & $\cdots$ & $\cdots$ & 1). & $\cdots$ & $\cdots$ & $\cdots$ \\
\hline \multicolumn{8}{|l|}{ MOLLUSCA } \\
\hline Abra alba & & $\cdots$ & $\cdots$ & I & $\ddot{i}$ & $\cdots$ & $\cdots$ \\
\hline Lutraria l & $\begin{array}{l}\text { aria } \\
\text { ta }\end{array}$ & $\cdots$ & $\cdots$ & $\cdots$ & 1). & $\ddot{r}$ & $\cdots$ \\
\hline Chione fas & & $\begin{array}{l}\cdots \\
\cdots\end{array}$ & $\begin{array}{l}\cdots \\
\cdots\end{array}$ & $\because$ & $\cdots$ & 1 & $\ddot{I}$ \\
\hline Cardium & & $\ldots$ & $\ddot{I}$ & $\begin{array}{l}\cdots \\
\cdots\end{array}$ & $\ddot{2}$ & $\cdots$ & 1 \\
\hline Aloidis git & & . & . & $\cdots$ & . & $\cdots$ & $\ddot{r}$ \\
\hline Cultellus 1 & $\begin{array}{l}\text { ucidus } \\
\text { mmunis }\end{array}$ & 4 & $t^{\star}$ & I & $\begin{array}{l}\cdots \\
\cdots\end{array}$ & $\cdots$ & I \\
\hline $\begin{array}{l}\text { Turritella } \\
\text { Aporrhais }\end{array}$ & $\begin{array}{l}\text { mmunis } \\
\text { s-pelicani }\end{array}$ & $\cdots$ & .. & $\ldots$ & $\cdots$ & $\cdots$ & $\begin{array}{l}1 \\
2\end{array}$ \\
\hline Philine ap & $a$ & I & $\cdots$ & $\cdots$ & $\cdots$ & $\cdots$ & 2 \\
\hline \multicolumn{8}{|l|}{ POLYZOA } \\
\hline Cellaria $\mathrm{s}$ & & $\cdots$ & $\cdots$ & I & $\cdots$ & + & I \\
\hline \multicolumn{8}{|c|}{ ECHINODERMATA } \\
\hline Amphiura & iformis & $\cdots$ & $\cdots$ & $\cdots$ & I & ? I & 2 \\
\hline Cucumari & longata & $\cdots$ & $\cdots$ & $\cdots$ & $\cdots$ & . & $\mathbf{I}^{*}$ \\
\hline
\end{tabular}

* One egg-cluster of Turritella. $\quad$ For abbreviations, see Table I. 


\section{Table III. Fauna of Mixed Muddy and Gravel Grounds in Teignmouth BAy (CONTINUED)}

Note

Station 9. 29. vii. 48. Exmouth Church, $349^{\circ}, 5 \cdot 3^{\prime}$. Coarse muddy sand and a few shell fragments. All sorted.

Station ro. 29. vii. 48. Exmouth Church, $349^{\circ}, 5^{\circ} 8^{\prime}$. Soil as at Station 9. One-tenth sorted. Station II. 29. vii. 48. Exmouth Church, $349^{\circ}, 6 \cdot 2^{\prime}$. Soil as at Stations 9 and IO. All sorted. Station I3. 30. vii. 48. Langstone Point, $334^{\circ}, 0.75^{\prime}$. Gravel and muddy sand. One-tenth sorted.

Station 23. 30. vii. 48 . Orestone, $222^{\circ}, 3 \cdot 5^{\prime}$. Muddy sand with Turritella shells. All sorted.

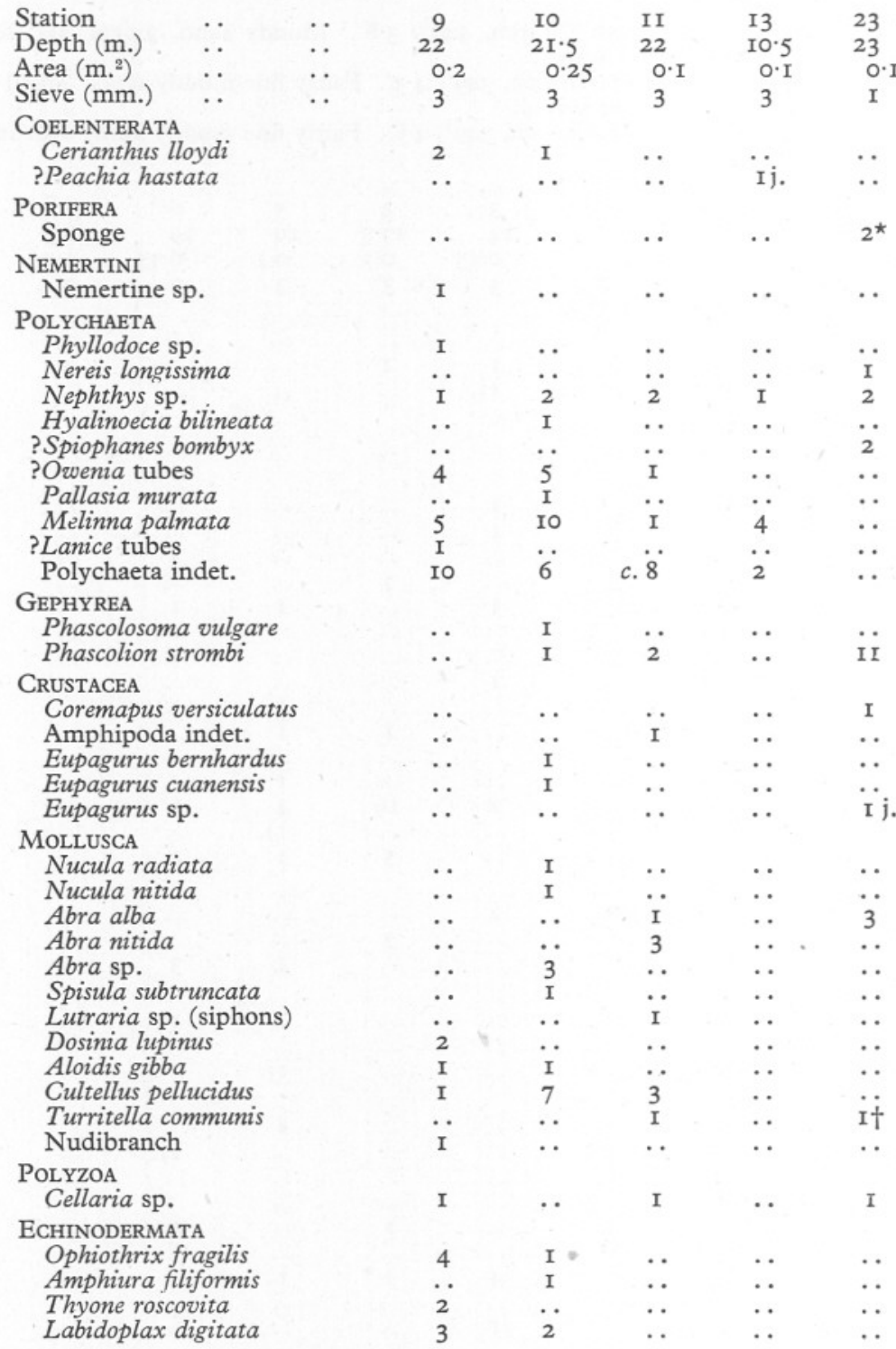

* Attached to Turritella shells. † Also c. 250 dead worn shells.

For abbreviations, see Table I. 


\section{Table IV. Fauna of Muddy Grounds in Teignmouth Bay}

Note

Station I7. 30. vii. 48. Langstone Point, or $7^{\circ}, \mathrm{I} \cdot \mathrm{I} 5^{\prime}$. Fine slightly muddy sand, and some gravel. Sampler not

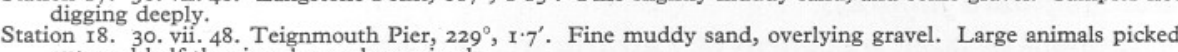
out, and half the sieved gravel examined.

Station 19. 30. vii. 48. Teignmouth Pier, $251^{\circ}, 1 \cdot 7^{\prime}$. Gravel and sand in first two hauls, last three of sand. Large ramined.

20. 30. vii. 48. Teignmouth Pier, $324^{\circ}, 0.6^{\prime}$. Fine sand with little mud.

Station 21. 30. vii. 48. Teignmouth Pier, $004^{\circ}, I^{\prime} 9^{\prime}$. Very fine muddy sand.

Station 22. 30 . vii. 48 . Orestone, $210^{\circ}, 2 \cdot 2^{\prime}$. Mud, with old Turritella shells.

\begin{tabular}{|c|c|c|c|c|c|c|c|}
\hline Station & . & I7 & I8 & I9 & 20 & $2 \mathrm{I}$ & 22 \\
\hline Depth (m.) & . & 10.5 & 12.5 & 16.5 & $9 \cdot 5$ & 10.5 & $2 I \cdot 5$ \\
\hline Area $\left(\mathrm{m} .^{2}\right)$ & $\cdots$ & 0.2 & 0.1 & 0.25 & 0.1 & 0.15 & $0 \cdot \mathrm{I}$ \\
\hline Sieve (mm.) & $\cdots$ & I & I & I & I & I & I \\
\hline CoELENTERATA & & & & & & & \\
\hline Cerianthus llo & & $\ddot{I}$ & $\because$ & 3 & $\cdots$ & $\because$ & $\because$ \\
\hline $\begin{array}{l}\text { ?Peachia hasta } \\
\text { Anemone }\end{array}$ & & $\begin{array}{l}1 \\
. .\end{array}$ & $\because$ & $\cdots$ & $\cdots$ & $\ddot{r}$ & $\cdots$ \\
\hline $\begin{array}{l}\text { Anemone } \\
\text { PolychaETa }\end{array}$ & & $\cdots$ & $\cdots$ & $\cdots$ & $\cdots$ & 1 & * \\
\hline Aphroditidae & inly polynoids) & I & . & I & $\cdots$ & I j. & $\cdots$ \\
\hline Sigalion math & & I & . & I & 2 & .. & $\because$ \\
\hline Sthenelais boa & & $\cdots$ & $\cdots$ & . & . & I & $\cdots$ \\
\hline Phyllodoce sp & & $\because$ & $\cdots$ & $\cdots$ & $\cdots$ & I & . \\
\hline ?Eteone sp. & & $\mathbf{I}$ & $\cdots$ & $\cdots$ & - & $\cdots$ & $\because$ \\
\hline $\begin{array}{l}\text { Nereis longissi } \\
\text { Nereid }\end{array}$ & & $\because$ & $\because$ & $\ddot{i}$ & $\because$ & $\cdots$ & Ij. \\
\hline & & $\ddot{6}$ & $\because$ & $\begin{array}{l}1) . \\
2\end{array}$ & $\because$ & c.s & 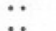 \\
\hline $\begin{array}{l}\text { Nephthys sp. } \\
\text { Glycera sp. }\end{array}$ & & .. & $\cdots$ & I & 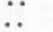 &.. & $\because$ \\
\hline Eone nordman & & * & $\mathbf{I}$ & I & . & ? & $\cdots$ \\
\hline Nematonereis & cornis & $\cdots$ & . & I & .. & . & $\cdots$ \\
\hline Lumbriconerei & & $\mathbf{I}$ & $\cdots$ & I & $\because$ & $\cdots$ & $\cdots$ \\
\hline Spionid & & $\cdots$ & $\cdots$ & $\mathbf{I}$ & $\mathbf{I}$ & $\ddot{x}$ & $\cdots$ \\
\hline Spiophanes bo & & $\ddot{i}$ & $\cdots$ & 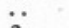 & $\because$ & c. IO & $\cdots$ \\
\hline Magelona pap & $\begin{array}{l}\text { cornis } \\
\text { ceus }\end{array}$ & I7 & $\cdots$ & 2 & 2 & I & $\ddot{i}$ \\
\hline Notomastus lo & ceus & $\ddot{z}$ & $\because$ & $\ddot{z}$ & $\ddot{2}$ & $\cdots$ & $2 j$. \\
\hline & & 2 & $\because$ & $+\star$ & 2 & $\because$ & $\ddot{*}$ \\
\hline $\begin{array}{l}\text { Pallasia mura } \\
\text { Pectinaria sp. }\end{array}$ & & $\therefore$ & $\ddot{I}$ & . & $\ldots$ & $\ddot{*}$ & $\ddot{m}$ \\
\hline Melinna palm & & 3. & . & 12 & $\mathrm{I}$ & c. 25 & $\ldots$ \\
\hline Amphitrite ed & $d s i$ & I j. & $\cdots$ & $\cdots$ & .. & . & . \\
\hline Lanice tubes & & $\ddot{0}$ & $\because$ & $\ddot{q}$ & 3 & $\ddot{B}$ & $\cdots$ \\
\hline Polychaeta in & & I8 & 5 & 7 & $\mathbf{I}$ & c. 20 & $\cdots$ \\
\hline GEPHYREA & & & & & & & \\
\hline Phascolion str & & $\cdots$ & I & $\cdots$ & $\cdots$ & $\cdots$ & $\cdots$ \\
\hline $\begin{array}{l}\text { CRUSTACEA } \\
\text { Iphinoë trispit }\end{array}$ & & & & & & & \\
\hline $\begin{array}{l}\text { Iphinoe trispi } \\
\text { Diastylis laev }\end{array}$ & & $\because$ & $\ddot{0}$ & 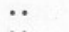 & $\cdots$ & 2 & $\cdots$ \\
\hline & rnis & $\because$ & 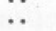 & $\because$ & $\because$ & $\begin{array}{l}1 \\
3\end{array}$ & $\ddot{q}$ \\
\hline $\begin{array}{l}\text { Ampelisca bre } \\
\text { Ampelisca ten }\end{array}$ & rnis & . & . & $\ddot{I}$ & $\ddot{x}$ & $\begin{array}{l}3 \\
. .\end{array}$ & $\because$ \\
\hline Bathyporeia s & & $\cdots$ & $\cdots$ & . & .. & $\ddot{2}$ & $\ddot{*}$ \\
\hline Urothöe grim & & $\cdots$ & $\cdots$ & .. & . & 2 & $\ddot{*}$ \\
\hline Amphipoda $\mathrm{i}$ & & $\because$ & $\cdots$ & $\cdots$ & 2 & . & $\because$ \\
\hline Eupagurus ber & rdus & r j. & $\cdots$ & $\cdots$ & . & $\cdots$ & Ij. \\
\hline MOLIUSCA & & & & & & & \\
\hline Nucula nitida & & $\cdots$ & $\cdots$ & $\cdots$ & $2 \mathrm{j}$. & 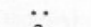 & $\cdots$ \\
\hline Thyasira flex & & $\ddot{\tau}$ & $\because$ & $\cdots$ & $\cdots$ & 3 & $\cdots$ \\
\hline Montacuta fer & inosa & $\begin{array}{l}\mathrm{I} \\
8\end{array}$ & $\because$ & $\because$ & $\cdots$ & $\because$ & $\cdots$ \\
\hline $\begin{array}{l}\text { Tellina fabuld } \\
\text { Abra alba }\end{array}$ & & $\begin{array}{l}8 \\
\cdots\end{array}$ & $\ddot{x}$ & $\because$ & $\cdots$ & 5 & $\cdots$ \\
\hline $\begin{array}{l}\text { Abra alba } \\
\text { Abra sp. }\end{array}$ & & $\cdots$ & $\ldots$ & 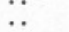 & $\ddot{I}$ & 7 & $\because$ \\
\hline Spisula subtru & & $\cdots$ & . & $\ldots$ & .. & $\ddot{I}$ & $\ddot{z}$ \\
\hline Lutraria sp. & 1ons) & 2 & $\cdots$ & I. & . & I & $\ldots$ \\
\hline Mysia undata & & $\because$ & . & $\mathrm{rj}$. & $\cdots$ & . & . \\
\hline Chione striatu & & I j. & . & $\because$ & I j. & * & $\cdots$ \\
\hline ?Mya truncata & & $\ddot{i}_{i}$ & $\cdots$ & I j. & $\cdots$ & * & $\cdots$ \\
\hline $\begin{array}{l}\text { Ensis sp. } \\
\text { Cultellus pellu }\end{array}$ & & $\begin{array}{l}\text { Ij. } \\
\text { Ij. }\end{array}$ & $\because$ & 4 & $\cdots$ & $\cdots$ & $\because$ \\
\hline Lamellibranc & det. & $\ldots$ & 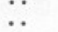 & $\because$ & $\ddot{0}$ & $\ddot{\mathrm{rj}}$. & $\ddot{*}$ \\
\hline Gibbula magu & & . & 2 & 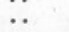 & $\ldots$ & . & $\because$ \\
\hline Turritella com & & ro & I & $\cdots$ & . & I & sot \\
\hline Retusa sp. & & $\cdots$ & $\cdots$ & $\cdots$ & $\cdots$ & I & $\cdots$ \\
\hline & & $\cdots$ & $\cdots$ & $\cdots$ & $\cdots$ & 2 & $\cdots$ \\
\hline ECHINODERMAT & & & & 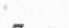 & & & \\
\hline Amphiura fili & $\begin{array}{l}\text { is } \\
\text { rdatum }\end{array}$ & I & $\mathbf{I}$ & 7 & $\mathbf{I}$ & 2 & .. \\
\hline Echinocardiun & & 1 & $\because$ & $\because$ & $\because$ & $\ddot{r}$ & $\cdots$ \\
\hline Leptosynapta & terens & I & $\because$ & $\ddot{I}$ & 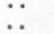 & $\begin{array}{l}1 \\
. .\end{array}$ & 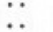 \\
\hline Labidoplax di & & I & . & . & $\cdots$ & .. & $\ldots$ \\
\hline
\end{tabular}


Outside the Turritella bed characteristic species are: Sigalion mathildae, Melinna palmata, Lutraria sp., Chione striatula, Ensis sp., Turritella communis and Amphiura filiformis.

Siphons of large specimens of Lutraria sp., probably L. lutraria, were taken at a number of stations. This mollusc digs to a considerable depth and the shell is out of reach of the 'bite' of the sampler.

\section{TORBAY}

Torbay was worked by Ford in 1923, but was not visited in 1948. Stations $\mathrm{F}_{3} \mathrm{I}-38$ (Table V) were worked, in a depth of II-I6 m. Torbay is sheltered on three sides, being open only to the eastward. The bottom is mostly fine muddy sand, and the fauna is rather similar to that off Teignmouth.

The main features are the presence of large numbers of Nucula nitida and Lutraria lutraria (?), the siphons only of the latter being taken in the Petersen grab. Ford notes that Echinocardium was more in evidence here than elsewhere in the Great West Bay.

Other typical species are: Nephthys sp., Melinna sp., Ampelisca sp., Thyasira flexuosa, Abra alba, Cultellus pellucidus and Amphiura filiformis.

\section{GROUNDS OFF BERRY HEAD}

Off Berry Head there is a deposit of very fine sand and mud. Stations $24-27$ (Table VI) were worked about 6 miles from shore, in a depth increasing from 30 to $5 \mathrm{I} \mathrm{m}$. The soil is similar at all stations except 27 , where it is rather coarser.

Characteristic species are: Nephthys sp., Lumbriconereis sp., Notomastus latericeus, Pectinaria koreni, Callianassa subterranea, ?Abra alba, Chione striatula, Cultellus pellucidus, Turritella communis (in small numbers), Amphiura filiformis, and Leptosynapta inhaerens. Callianassa occurs at all stations from 25 to 29, and evidently forms a bed of considerable density in this area. The average density is 13 per $\mathrm{m} .{ }^{2}$

\section{'THE CORNER'}

South of Station 27 the bottom becomes coarser and less muddy. Several species seem to reach a larger size than at previous stations. The area is trawled by Brixham fishing vessels, but is not very productive.

Stations 28-33 were worked in this area in a depth of 5I-60 m. (Table VII). The bottom is of coarse slightly muddy sand, characteristic species being: hydroids, Pectinaria koreni, Nucula nitida, Amphiura filiformis and Echinocyamus pusillus.

Echinocyamus appears for the first time at Station 28, not having been taken at previous stations. Station 33 has a bottom of finer sand with a fauna similar to that found on similar soils in Teignmouth Bay. 


\section{Table V. Fauna of Torbay. Sampled by Mr Ford in ig23 with two Hauls of the $\frac{1}{10} M .^{2}$ Petersen Grab at each Station}

Note

Station F 3I. I5. xi. 23. Saltern Cavern NW, Churston Point, SW by W $\frac{1}{2} W$. Sand.

Station F 32. I5. xi. 23. Red Cliff Hotel NW by N; Roundham Head NW by W $\frac{1}{2} W$. Silty sand.

Station F 33. I5. xi. 23. Orestone E by N; Torquay Pier N by W. Muddy sand.

Station F 34. I5. xi. 23. Orestone NE by N; Thatcher Rock N. Mud and sand.

Station F 35. I5. xi. 23. Berry Head S; Brixham Breakwater Light SW by W. Mud and sand.

Station F 36. I5. xi. 23. Berry Head SE; Brixham Breakwater Light WSW. Sand harder and cleaner than at Station 35.

Station F 37. I 5. xi. 23. Old Battery SW; Brixham Breakwater Light SE by E. Sandy mud. Station F 38. I5. xi. 23. Quarries WNW; Fishcombe Point S $\frac{1}{2}$ E. Mud, with some Zostera.

\begin{tabular}{|c|c|c|c|c|c|c|c|c|}
\hline $\begin{array}{l}\text { Station } \\
\text { Sounding (m.) }\end{array}$ & $\underset{\text { II }}{\mathrm{F}_{3 I}}$ & $\begin{array}{c}\mathrm{F}_{32} \\
\text { I5 }\end{array}$ & $\begin{array}{c}\mathrm{F}_{33} \\
\mathrm{I} 4\end{array}$ & $\begin{array}{c}\mathrm{F}_{34} \\
\mathrm{I} 6\end{array}$ & $\begin{array}{c}\mathrm{F}_{35} \\
\mathrm{I} 5\end{array}$ & $\begin{array}{l}\mathrm{F}_{36} \\
\mathrm{I} 2\end{array}$ & $\begin{array}{l}\mathrm{F}_{37} \\
\mathrm{I} 2\end{array}$ & $\begin{array}{c}\mathrm{F}_{38} \\
?\end{array}$ \\
\hline \multicolumn{9}{|l|}{ COELENTERATA } \\
\hline $\begin{array}{l}\text { Anemone } \\
\text { PLATYHELMINTHES }\end{array}$ & $\cdots$ & $\cdots$ & $\cdots$ & $\cdots$ & $\cdots$ & $\cdots$ & I & . \\
\hline \multicolumn{9}{|l|}{ Platyhelminthes } \\
\hline \multirow{2}{*}{\multicolumn{7}{|c|}{ Nemertini }} & $\cdots$ & I \\
\hline Nemertine sp. & . & I & I & $\cdots$ & I & & & \\
\hline \multicolumn{9}{|l|}{ Polychaeta } \\
\hline Sigalion sp. . . & . & . & . & . & $\cdots$ & . & I & $\cdots$ \\
\hline Sthenelais limicola & . & I & . & . & . & . & I & 2 \\
\hline Phyllodoce maculata & $\cdots$ & $\cdots$ & $\cdots$ & $\cdots$ & I & $\cdots$ & $\cdots$ & $\cdots$ \\
\hline Nereis sp. & $\because$ & I & $\because$ & $\because$ & $\because$ & . & $\cdots$ & $\cdots$ \\
\hline Nephthys sp. & 6 & IO & 8 & 6 & 8 & 7 & 7 & 9 \\
\hline Ammotrypane sp. & $\cdots$ & I & $\cdots$ & $\cdots$ & $\cdots$ & . & I & $\because$ \\
\hline Scalibregma sp. & $\cdots$ & $\because$ & $\cdots$ & $\cdots$ & $\cdots$ & $\cdots$ & $\cdots$ & I \\
\hline Notomastus latericeus & $\cdots$ & I & $\cdots$ & $\cdots$ & $\cdots$ & $\cdots$ & $\cdots$ & $\cdots$ \\
\hline $\begin{array}{l}\text { Pectinaria auricoma } \\
\text { Melinna } \mathrm{sp} \text {. }\end{array}$ & $\ddot{+}$ & I & $\ddot{3}$ & $\ddot{2}$ & $\cdots$ & $\begin{array}{l}\cdots \\
\cdots\end{array}$ & $\ddot{+}$ & $\ddot{+}$ \\
\hline $\begin{array}{l}\text { Melinna sp. } \\
\text { Lanice conchilega tubes }\end{array}$ & $\begin{array}{l}+ \\
3\end{array}$ & 5 & $\begin{array}{l}3 \\
\mathrm{I}\end{array}$ & . & $\cdots$ & $\ddot{6}$ & . & 2 \\
\hline Polychaeta indet. & + & $\ddot{+}$ & + & 6 & + & $\ldots$ & + & + \\
\hline \multicolumn{9}{|l|}{ CRUSTACEA } \\
\hline Diastylis sp. & & 2 & I & . & . & . & . & . \\
\hline Ampelisca sp. & 6 & I & 3 & 4 & $\cdots$ & . & 2 & I \\
\hline ? Maera sp. & I & . & . & . & $\cdots$ & $\cdots$ & $\because$ & $\cdots$ \\
\hline Amphipoda indet. & $\cdots$ & $\cdots$ & $\cdots$ & $\cdots$ & $\cdots$ & $\cdots$ & I j. & $\cdots$ \\
\hline Caprellid & $\because$ & $\cdots$ & . & . & . & $\cdots$ & $\cdots$ & I \\
\hline Porcellana longicornis & I & $\cdots$ & $\cdots$ & $\cdots$ & . & $\cdots$ & $\cdots$ & $\ddot{i}$ \\
\hline Macropodia sp. & $\cdots$ & $\cdots$ & $\cdots$ & $\cdots$ & $\cdots$ & $\cdots$ & $\cdots$ & 21. \\
\hline \multicolumn{9}{|l|}{ MOLLUSCA } \\
\hline Nucula nitida & 7 & 25 & 9 & I2 & 2 & 3 & 4 & 22 \\
\hline Thyasira flexuosa & $\cdots$ & 6 & I & $\cdots$ & $\cdots$ & $\because$ & I & 5 \\
\hline Mysella bidentata & . & 3 & . & $\cdots$ & $\cdots$ & I & $\cdots$ & 2 \\
\hline Montacuta ferruginosa & $\cdots$ & $\cdots$ & . & $\because$ & 4 & $\cdots$ & $\cdots$ & $\because$ \\
\hline Tellina fabula & 5 & 3 & $\cdots$ & I & $\cdots$ & $\cdots$ & $\cdots$ & 6 \\
\hline Abra alba & $\cdots$ & $\because$ & $3 \mathrm{j}$. & $4 \mathrm{j}$. & 29 & . & I & $6 \mathrm{j}$. \\
\hline Abra sp. & $\because$ & 71. & . & $\cdots$ & $\cdots$ & $\cdots$ & . & $\cdots$ \\
\hline Mactra corallina & I $j$ & $\cdots$ & . & . & $\cdots$ & . & . & . \\
\hline ? Spisula elliptica & . & $\cdots$ & $\cdots$ & I $)$. & $\cdots$ & $\cdots$ & . & $\cdots$ \\
\hline ?Lutraria lutraria (siphons) & $\cdots$ & I & $\cdots$ & $\cdots$ & $25^{\star}$ & I & $\cdots$ & $\cdots$ \\
\hline Chione striatula & . & I & .. & 2 & . & .. & 2 & $\cdots$ \\
\hline Cardium echinatum & . & . & . & $\cdots$ & 2 & . & $\cdots$ & . \\
\hline Aloidis gibba & $\cdots$ & $\cdots$ & .. & .. & . & $\cdots$ & I j. & .. \\
\hline Cultellus pellucidus & I & I & . & 9 & .. & . & . & 2 \\
\hline Philine sp. & $\cdots$ & $5 \mathrm{j}$. & $\cdots$ & I & . & .. & $\cdots$ & $4 j$ \\
\hline \multicolumn{9}{|l|}{ ECHINODERMATA } \\
\hline Astropecten sp. & $\cdots$ & $\cdots$ & $\cdots$ & $\cdots$ & I & $\cdots$ & $\cdots$ & . \\
\hline Amphiura filiformis & I & I3 & + & $\cdots$ & 7 & $\cdots$ & 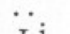 & . \\
\hline Ophiuroid & I & $\ddot{r}$ & $\ddot{v}$ & 2 & $\ddot{i} i$ & $\cdots$ & I 1. & $\cdots$ \\
\hline Echinocardium cordatum & . & I & 1 & $\cdots$ & 131. & $\cdots$ & $\cdots$ & $\cdots$ \\
\hline
\end{tabular}

* In all but four specimens siphons only were taken.

For abbreviations, see Table I. 
Table VI. Fauna of Grounds off Berry Head. Soll: Fine Sand and Mud at all Stations

\section{Note}

Station 24. 4. viii. 48. Berry Head, $225^{\circ}, 6 \cdot 2^{\prime}$.

Station 25. 4. viii. 48. Berry Head, 254 , 5. $7^{\prime}$.

Station 26. 4. viii. 48 . Berry Head, $280^{\circ}, 5^{\circ} 8^{\prime}$.

Station 27. 4. viii. 48. Berry Head, $297^{\circ}, 6 \cdot 4^{\prime}$.

\begin{tabular}{|c|c|c|c|c|}
\hline Station & 24 & 25 & 26 & 27 \\
\hline Depth (m.) .. & $30 \cdot 5$ & 40.5 & 48 & $5 \mathrm{I}$ \\
\hline Area $\left(\mathrm{m} .^{2}\right)$ & $0 \cdot 1$ & 0.1 & 0.15 & 0.25 \\
\hline Sieve (mm.) $\quad \ldots \quad \ldots$ & I & I & II & I \\
\hline \multicolumn{5}{|l|}{ COELENTERATA } \\
\hline Hydroids & . & . & + & + \\
\hline ?Sagartia sp. & 9 & $\cdots$ & . & I \\
\hline \multicolumn{5}{|l|}{ NEMERTINEA } \\
\hline Nemertine sp. & . & I & I3 & 2 \\
\hline \multicolumn{5}{|l|}{ Polychaeta } \\
\hline Aphroditidae & . & . & I & I \\
\hline ?Harmothoë lunulata & . & . & I & . \\
\hline Phyllodoce sp. & $\cdots$ & I & I & 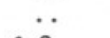 \\
\hline Nephthys sp. & $\cdots$ & 3 & 6 & c. 3 \\
\hline Glycera convoluta & $\cdots$ & $\cdots$ & $\ddot{i}$ & 3 \\
\hline Glycera sp. & $\cdots$ & $\ddot{r}$ & $\begin{array}{l}\text { I j). } \\
\text { I }\end{array}$ & * \\
\hline $\begin{array}{l}\text { ?Eone } \mathrm{sp} \text {. } \\
\text { Lumbriconereis } \mathrm{sp} \text {. }\end{array}$ & $\ddot{I}$ & $\begin{array}{l}\mathrm{I} \\
\ldots\end{array}$ & & $\ddot{2}$ \\
\hline $\begin{array}{l}\text { Lumbriconereis sp. } \\
\text { Scoloplos armiger }\end{array}$ & $\ldots$ & $\ddot{I}$ & $\begin{array}{l}2 \\
2\end{array}$ & $\begin{array}{l}2 \\
\cdots\end{array}$ \\
\hline Spiophanes bombyx & I & . & $? 2$ & $\because$ \\
\hline Cirratulid & . & 2 & 6 & $\because$ \\
\hline ?Stylaroides flabellata & $\cdots$ & . & 2 & . \\
\hline Scalibregma inflatum & I & . & .. & $\cdots$ \\
\hline Scalibregma sp. & $\because$ & 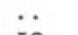 & $\because$ & I \\
\hline Notomastus latericeus & 3 & IO & 3 & I \\
\hline ?Owenia tubes & $\ddot{r}$ & . & $\because$ & 2 \\
\hline Pectinaria koreni & I & $\ddot{\dot{p}}$ & 4 & 22 \\
\hline Terebellid & $\cdots$ & $? 3 \mathrm{j}$ & 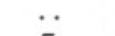 & I \\
\hline Polychaeta indet. & $\cdots$ & II & 7 & 4 \\
\hline \multicolumn{5}{|l|}{ Crustacea } \\
\hline $\begin{array}{l}\text { Diastylis sp. } \\
\text { Cumacean }\end{array}$ & $\cdots$ & . & $\cdots$ & I \\
\hline $\begin{array}{l}\text { Cumacean } \\
\text { Ampelisca tenuicornis }\end{array}$ & $\ddot{x}$ & $\cdots$ & I & .. \\
\hline $\begin{array}{l}\text { Ampelisca tenuicornis } \\
\text { Harpinia antennaria }\end{array}$ & I & I & I & . \\
\hline $\begin{array}{l}\text { Harpinia antennaria } \\
\text { Callianassa subterranea }\end{array}$ & $\cdots$ & & 2 & $\ddot{i}$ \\
\hline $\begin{array}{l}\text { Callianassa subterranea } \\
\text { Decapoda }\end{array}$ & $\begin{array}{l}\cdots \\
.\end{array}$ & 2 & $2+4 \mathrm{j}$. & $2 \mathrm{j}$. \\
\hline $\begin{array}{l}\text { Decapoda } \\
\text { MolluSCA }\end{array}$ & \multicolumn{2}{|c|}{ MOLLUSCA } & 2 & $\cdots$ \\
\hline MOLLUSCA & . & $\cdots$ & * & 5 \\
\hline ? Abra alba & . & Io j. & $4 \mathrm{j}$. & $27 \mathrm{j}$. \\
\hline $\begin{array}{l}\text { ?Dosinia exoleta } \\
\text { ?Dosinia lupinus }\end{array}$ & $\cdots$ & $\cdots$ & . & I. \\
\hline ?Dosinia lupinus & $\cdots$ & . & $\ddot{s}$ & I j. \\
\hline $\begin{array}{l}\text { Chione striatula } \\
\text { Cardium aculeatum }\end{array}$ & $\cdots$ & $\cdots$ & $3 \mathrm{j}$. & $\mathrm{I}+6 \mathrm{j}$ \\
\hline $\begin{array}{l}\text { ?Cardium aculeatum } \\
\text { Aloidis gibba }\end{array}$ & $\cdots$ & $\cdots$ & $\ddot{i}$ & I j. \\
\hline $\begin{array}{l}\text { Aloidis gibba } \\
\text { Cultellus pellucidus }\end{array}$ & $\ddot{i j}$. & $\ddot{\mathrm{i}}$ & 31. & 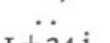 \\
\hline $\begin{array}{l}\text { Cultellus pellucidus } \\
\text { Psammosolen chamasolen }\end{array}$ & 1). & $\begin{array}{l}\text { I }) . \\
\ldots\end{array}$ & $\ddot{I}$ & $\mathrm{I}+24 \mathrm{j}$. \\
\hline $\begin{array}{l}\text { Psammosolen chamasolen } \\
\text { Lamellibranch indet. }\end{array}$ & $\because$ & $\ddot{m}$ & $\begin{array}{l}\text { I } \\
\text { I }\end{array}$ & . \\
\hline $\begin{array}{l}\text { Lamellibranch indet. } \\
\text { Turritella communis }\end{array}$ & $12 *$ & $\ddot{+}+$ & $\begin{array}{l}1 \\
+t\end{array}$ & $\cdots$ \\
\hline & . & I & $\begin{array}{l}+\mathrm{T} \\
\cdots\end{array}$ & $\cdots$ \\
\hline \multicolumn{5}{|l|}{ ECHINODERMATA } \\
\hline Amphiura filiformis & . & . & $10 \mathrm{j}$. & $5 \mathrm{I}$ \\
\hline Echinocardium cordatum & . & . & . & 2 \\
\hline Leptosynapta inhaerens & $\ldots$ & .. & I & I2 \\
\hline
\end{tabular}




\section{Table VII. Fauna of 'The Corner' Region}

\section{Note}

Station 28. 4. viii. 48. Berry Head, $310^{\circ}, 8 \cdot 2^{\prime}$. Muddy sand with shell fragments. Soil coarser and less muddy than at Station 27.

Station 29. 4. viii. 48 . Berry Head, $319^{\circ}, 9 \cdot 4^{\prime}$. Coarse slightly muddy sand. One-quarter sorted after picking out large animals.

Station 30. 4. viii. 48 . Start Point, $275^{\circ}, 9 \cdot 6^{\prime}$. Coarse slightly muddy sand, and shell fragments. Sampler not bringing up much soil.

Station 3I. 4. viii. 48. Start Point, $282^{\circ}, 6 \cdot 3^{\prime}$. Coarse slightly muddy sand. One-quarter sorted.

Station 32. 4. viii. 48. Start Point, $270^{\circ}, 5 \cdot 4^{\prime}$. Coarse slightly muddy sand. One-quarter sorted.

Station 33. 4. viii. 48. Start Point, $240^{\circ}, 5 \cdot 6^{\prime}$. Slightly muddy sand.

\begin{tabular}{|c|c|c|c|c|c|c|}
\hline Station & 28 & 29 & 30 & $3 I$ & 32 & 33 \\
\hline Depth (m.) & $5 \mathrm{I}$ & 54.5 & 60 & 59.5 & $58 \cdot 5$ & 49 \\
\hline Area $\left(\mathrm{m} \cdot{ }^{2}\right)$ & 0.25 & 0.15 & $0 \cdot 1$ & 0.1 & 0.15 & 0.2 \\
\hline Sieve (mm.) & 2 & 2 & I & I & 2 & $?$ \\
\hline \multicolumn{7}{|l|}{ COELENTERATA } \\
\hline Hydroids & I & $\mathbf{I}$ & + & $\cdots$ & ++ & $\cdots$ \\
\hline Cerianthus lloydi & . & . & $\cdots$ & $\cdots$ & 4 & . \\
\hline \multicolumn{7}{|l|}{ NEMERTINEA } \\
\hline Nemertine & . & 2 & . & . & . & . \\
\hline \multicolumn{7}{|l|}{ Polychaeta } \\
\hline Aphroditidae & - & I & . & . & . & I \\
\hline Sigalion mathildae & . & . & $\cdots$ & $\cdots$ & . & I \\
\hline Sthenelais limicola & . & . & $\cdots$ & . & $\cdots$ & I \\
\hline Phyllodocidae & $\cdots$ & $\cdots$ & $\cdots$ & $\cdots$ & $\ddot{i}$ & I \\
\hline $\begin{array}{l}\text { Nereidae } \\
\text { Nephthys sp. }\end{array}$ & c. $\ddot{3}$ & $\begin{array}{l}\cdots \\
\ldots\end{array}$ & 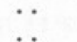 & $\because$ & I). & c. $\ddot{6}$ \\
\hline $\begin{array}{l}\text { Nephthys sp. } \\
\text { Glycera convoluta }\end{array}$ & $\begin{array}{l}\text { C. } 3 \\
\ldots\end{array}$ & $\ddot{I}$ & $\ddot{I}$ & $\begin{array}{l}\cdots \\
\cdots\end{array}$ & $\begin{array}{l}\cdots \\
\cdots\end{array}$ & $\begin{array}{l}\text { c. } 0 \\
. .\end{array}$ \\
\hline $\begin{array}{l}\text { Glycera convoluta } \\
\text { Glycera sp. }\end{array}$ & . & . & . & . & $\cdots$ & $\ddot{3}$ \\
\hline & I & * & . & $\cdots$ & . & 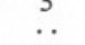 \\
\hline Lumbriconereis sp. & $\cdots$ & 2 & I & $\cdots$ & $\ldots$ & 5 \\
\hline Magelona papillicornis & $\cdots$ & . & I & $\cdots$ & . & 160 \\
\hline Scalibregma inflatum & 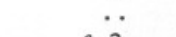 & $\cdots$ & . & $\cdots$ & $\cdots$ & I \\
\hline ?Owenia tubes & c. 3 & 2 & I & . & I & . \\
\hline Pallasia murata. & I & $\cdots$ & . & $\because$ & $\because$ & $\ldots$ \\
\hline Pectinaria koreni & IO & 4 & $\cdots$ & I & I & I3 \\
\hline Pectinaria auricoma & I & $\cdots$ & . & $\cdots$ & $\because$ & $\cdots$ \\
\hline ?Lanice tubes & . & 4 & . & $\cdots$ & I & $\ldots$ \\
\hline Dasychone bombyx & $\cdots$ & $\because$ & $\because$ & $\cdots$ & I & $\cdots$ \\
\hline Terebellid & $\ddot{m}$ & I & I & $\cdots$ & $\cdots$ & $\ddot{2}$ \\
\hline Polychaeta indet. & IO & 6 & 2 & 4 & $\cdots$ & c. 20 \\
\hline \multicolumn{7}{|l|}{ CRUSTACEA } \\
\hline Ampelisca spinipes & $\because$ & $\cdots$ & $\ddot{z}$ & I & $\ddot{I}$ & $\cdots$ \\
\hline Caprellid & $\ddot{r}$ & $\ddot{I}_{j}$. & $\begin{array}{l}\cdots \\
. .\end{array}$ & 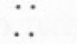 & $\begin{array}{l}1 \\
.\end{array}$ & $\because$ \\
\hline $\begin{array}{l}\text { Callianassa subterranea } \\
\text { Upogebia stellata }\end{array}$ & $\begin{array}{l}\mathrm{I} \\
\cdots\end{array}$ & $\begin{array}{l}\text { I). } \\
\cdots\end{array}$ & . & I & $\cdots$ & $\ddot{m}$ \\
\hline Corystes cassivelaumus & I & . & . & . & . & $\ddot{2}$ \\
\hline \multicolumn{7}{|l|}{ MOLLUSCA } \\
\hline Chiton & $\cdots$ & $\cdots$ & $\because$ & $\cdots$ & I & $\cdots$ \\
\hline Nucula nitida & I & I & Ij. & $\cdots$ & . & 4 \\
\hline Diplodonta rotundata & . & $\cdots$ & $\cdots$ & I & . & $\cdots$ \\
\hline Tellina donacina & $\cdots$ & $\cdots$ & $\cdots$ & I & $\ddot{i}$ & $\ddot{i s}$ \\
\hline $\begin{array}{l}\text { Abra alba } \\
\text { Abra nitida }\end{array}$ & $\ddot{2}$ & $\ddot{2}$ & $\therefore$ & $\because$ & I0 & I5 \\
\hline $\begin{array}{l}\text { ?Abra nitida } \\
\text { Abra sp. }\end{array}$ & . & . & $\ddot{I}$ & $\begin{array}{l}\cdots \\
\ldots\end{array}$ & $\begin{array}{l}\cdots \\
\cdots\end{array}$ & $\begin{array}{l}\cdots \\
\cdots\end{array}$ \\
\hline $\begin{array}{l}\text { Abra sp. } \\
\text { Gari ferroensis }\end{array}$ & I & . & . & . & . & $\ddot{\cdots}$ \\
\hline Dosinia lupinus & I & I & . & . & $\ldots$ & $\cdots$ \\
\hline Chione striatula & 2 & $\cdots$ & . & . & $\cdots$ & I \\
\hline Cardium sp. & $\cdots$ & $\because$ & . & . & . & rj. \\
\hline Aloidis gibba & . & I & . & . & . & $\because$ \\
\hline Ensis sp. & $\cdots$ & $\cdots$ & . & . & $\because$ & Ij. \\
\hline Cultellus pellucidus & 3 & . & $\cdots$ & . & I & $3 \mathrm{j}$. \\
\hline Lamellibranch indet. & $\cdots$ & $\cdots$ & . & 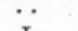 & $\cdots$ & I \\
\hline Dentalium sp. & $\cdots$ & $\cdots$ & $\cdots$ & I & $\cdots$ & $\ddot{i t}$ \\
\hline Turritella communis & $\cdots$ & $\cdots$ & $\cdots$ & . & $\cdots$ & $+t$ \\
\hline \multicolumn{7}{|l|}{ ECHINODERMATA } \\
\hline Amphiura filiformis & 33 & 7 & $\cdots$ & I & 3 & 6 \\
\hline $\begin{array}{l}\text { Ophiura texturata } \\
\text { Echinocvamus pusillus }\end{array}$ & $\ddot{+} \star$ & $\ddot{+*}$ & $\ddot{+}$ * & $\ddot{t^{*}}$ & $\ddot{4 *}$ & I \\
\hline Echinocyamus pusillus & $T$ & $T$ & $T$ & $T$ & $4^{n}$ & $\cdots$ \\
\hline
\end{tabular}




\section{START BAY}

A mile or so off Slapton the bottom is of fine muddy sand, with the following fauna:

Station 34. 5. viii. 48. Start Point $188^{\circ}, 3 \cdot 2^{\prime}$. Fine slightly muddy sand. Depth $\mathrm{r} 8 \mathrm{~m}$. O. I m.2 sampled. Sieve: I mm.

Phyllodocidae
Magelona papillicornis
Polychaeta indet.
Nucula nitida
Abra alba

$\begin{array}{rll}\text { I } & \text { ? Mysella bidentata } & 2 \\ \text { I } & \text { Montacuta ferruginosa } & \text { I } \\ \text { I3 } & \text { Ensis sp. } & \text { I } \\ 3 & \text { Cultellus pellucidus } & 2 \\ 9 & \text { Natica catena } & \text { I }\end{array}$

Closer in-shore a gravel bottom occurs, with Spisula solida as the main inhabitant. This ground has already been described by Ford (1925), who found that the bed of Spisula extended for about 4 miles along the coast, in a depth of about $10 \mathrm{~m}$. The density exceeded 1000 per $\mathrm{m} .{ }^{2}$ in six out of nine stations. In 1948 Stations 35-37 were worked on the Spisula bed. The results may be summarized thus:

$\begin{array}{lll}\text { Station } 35 & \text { O. I m. } .^{2} \text { sampled } & \begin{array}{l}\text { Empty Spisula shells only } \\ \text { Station } 36\end{array} \\ \text { O.15 m. } .^{2} \text { sampled } & \begin{array}{l}\text { Spisula solida } 58 \\ \text { Chione fasciata } \mathrm{I}\end{array} \\ \text { Station } 37 & 0.05 \mathrm{~m} .^{2} \text { sampled } & \text { Empty Spisula } \text { shells only }\end{array}$

Bearings and distances from Start Point were:

$$
\text { 35: } 181^{\circ}, 3.3^{\prime} ; 36: 181^{\circ}, 3 \cdot 5^{\prime} ; 37: 182^{\circ}, 3 \cdot 7^{\prime} \text {. }
$$

Dredge hauls confirmed the abundance of Spisula, although it may be noted that the density recorded, 387 per m. ${ }^{2}$, is considerably less than all but one (no. 50) of the nine stations sampled by Ford.

\section{COMPARISON WITH FoRD's SURVEY}

The results of Ford's survey are given in Tables V, VIII, IX and X. From these it is clear that no major qualitative changes have occurred in the fauna. For example, the following were found in both surveys:

(I) The Turritella ground in Teignmouth Bay.

(2) The Spisula bed in Start Bay.

(3) A fauna with Nephthys sp., Notomastus latericeus, Melinna palmata, Nucula nitida, Lutraria sp., Cultellus pellucidus and Amphiura filiformis among the most abundant or widely distributed species.

Owing to the different types of sampler used and to the fact that the same stations were not revisited, a quantitative comparison of the fauna at the two different times is not possible. The Spisula bed may have declined in numbers, as shown above, and certain species, Abra alba for example, seem to have decreased in density. On the whole, however, there is no evidence of any overall change in the abundance of the fauna. 


\title{
TABle VIII. SAmples taken by Mr Ford in the TURRItella Bed iN Teignmouth Bay. Two Hauls of $\frac{1}{10}$ M. ${ }^{2}$ Petersen Grab at Each STATION
}

\author{
Note
}

Station F 14. 13. xi. 23. Hope Nose W $\frac{1}{2} S$, The Ness NW by W. Mud and Turritella shells. Sample with over 700 dead and worn Turritella shells.

Station F I7. 13. xi. 23. Orestone $\mathrm{S}_{\frac{1}{2}} \mathrm{~W}$, Babbacombe Point $\mathrm{W}$ by $\mathrm{S}_{2} \mathrm{~S}$. Black mud and brownish sand. Ten empty Turritella shells taken.

Station F I8. I3. xi. 23. Babbacombe Point SW $\frac{1}{2} S$, The Ness N $\frac{1}{2} E$. Dark sand and mud. Thirty empty shells of Turritella, but these were fresh and pink in colour.

Station F 20. I3. xi. 23. Orestone S by W, The Ness NW. Muddy shell gravel with Turritella shells. Over 600 dead and worn shells taken.

Station F 23. I4. xi. 23. Orestone NW by W $\frac{1}{2} W$, The Ness $N$ by W $\frac{1}{4} W$. Dark sandy mud and Turritella shells. Over I 200 white empty shells taken, but these were not badly worn.

\begin{tabular}{|c|c|c|c|c|c|}
\hline $\begin{array}{l}\text { Station } \\
\text { Sounding }(\mathrm{m} .)\end{array}$ & $\begin{array}{c}\mathrm{F}_{14} \\
30\end{array}$ & $\begin{array}{l}\mathrm{F}_{17} \\
\text { I9 }\end{array}$ & $\begin{array}{l}\text { Fr8 } \\
\text { I7 }\end{array}$ & $\begin{array}{c}\mathrm{F}_{2} \mathrm{O} \\
2 \mathrm{I}\end{array}$ & $\begin{array}{c}\mathrm{F}_{23} \\
32\end{array}$ \\
\hline \multicolumn{6}{|l|}{ COELENTERATA } \\
\hline $\begin{array}{l}\text { Hydroid } \\
\text { Anemone }\end{array}$ & $\begin{array}{l}\text { I } \\
\text { I }\end{array}$ & $\ddot{\mathrm{I}}$ &.. & $\because$. & $\ddot{2}$ \\
\hline \multicolumn{6}{|l|}{ PLATYHELMINTHES } \\
\hline Cryptocoelis sp. & I & .. & . & .. & .. \\
\hline \multicolumn{6}{|l|}{$\begin{array}{l}\text { PolychaETA } \\
\text { Aphrodite sp. }\end{array}$} \\
\hline $\begin{array}{l}\text { Aphrodite sp. } \\
\text { Aphroditidae }\end{array}$ & $\ddot{I}$ & $\therefore$ & $\because$ & $\begin{array}{l}\mathrm{I} \\
. .\end{array}$ & $\because$ \\
\hline Sthenelais sp. & I & .. & .. & ... & $\ddot{x}$ \\
\hline Nephthys sp. & 3 & 22 & 2 & 2 & 4 \\
\hline Goniada maculata & . & I & . & $\ddot{\sigma}$ & .. \\
\hline Scalibregma sp. & . & . & $\because$ & I & .. \\
\hline Ammotrypane sp. & .. & . & I & .. & .. \\
\hline Notomastus sp. & + & + & .. & .. & .. \\
\hline Owenia fusiformis & . & I & .. & ro & .. \\
\hline Melinna sp. & $\cdots$ & + & 4 & 13 & 5 \\
\hline Lanice sp. & .. & .. & $\cdots$ & I & . \\
\hline Terebellid & .. & I & .. & .. & .. \\
\hline Sabellid & .. & $\cdots$ & .. & I & 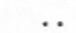 \\
\hline Polychaeta indet. & I & + & .. & + & + \\
\hline \multicolumn{6}{|l|}{ GEPHYREA } \\
\hline Phascolion strombi & + & . & 9 & .. & + \\
\hline \multicolumn{6}{|l|}{ CRUSTACEA } \\
\hline $\begin{array}{l}\text { Ampelisca sp. } \\
\text { Amphipoda indet. }\end{array}$ & $\begin{array}{l}\text { I } \\
\text { I }\end{array}$ & I & $\because$. & $\begin{array}{l}3 \\
I\end{array}$ & $\ddot{0}$ \\
\hline $\begin{array}{l}\text { Amphipoda indet. } \\
\text { Shrimp }\end{array}$ & .. & $\ddot{I}$ & .. & .. & $\ddot{x}$ \\
\hline Eupagurus sp. & $7 \mathrm{j}$. & . & .. & 6 & . \\
\hline Nika edulis & $\therefore$ & I & .. & $\ldots$ & $\ddot{\tau}$ \\
\hline $\begin{array}{l}\text { Gonoplax rhomboides } \\
\text { Porcellana longicornis }\end{array}$ & $\ddot{8}$ & 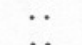 & $\because$ & $\ddot{I}$ & $\mathrm{I}$ \\
\hline \multicolumn{6}{|l|}{ MollusCa } \\
\hline Thyasira flexuosa & I & 3. & .. & 3 & $\cdots$ \\
\hline Abra alba & ?25 & $2 \mathrm{j}$. & .. & 7 & . \\
\hline Mactra corallina & .. & $\mathrm{Ij}$. & .. & & .. \\
\hline Aloidis gibba & . & .. & .. & I & \\
\hline $\begin{array}{l}\text { Cultellus pellucidus } \\
\text { Bullinellavindrat }\end{array}$ & . & $\cdots$ & $\ddot{I}$ & I & $\cdots$ \\
\hline $\begin{array}{l}\text { Bullinella cylindracea } \\
\text { Nassa reticulata }\end{array}$ & $\because$. & $\because$. & $\begin{array}{l}1 \\
1\end{array}$ & $\begin{array}{l}. . \\
\end{array}$ & \\
\hline Turritella communis & $\ddot{58}$ & . & 70 & .. & $3^{8}$ \\
\hline \multicolumn{6}{|l|}{ ECHINODERMATA } \\
\hline Ophiuroid & .. & I j. & .. & $\ldots$ & . \\
\hline
\end{tabular}

For abbreviations, see Table I. 


\section{Table IX. Samples taken by Mr Ford on Various Grounds in Teign- mouth Bay. Two Hauls of the $\frac{1}{10}$ M. ${ }^{2}$ Petersen Grab at Each Station \\ Note}

Station F I5. 13. xi. 23. Hope Nose S, Babbacombe Point W by S. Dark mud and sand.

Station F I6. 13. xi. 23. Babbacombe Point S, Petit Tor NW. Fine red sand.

Station F 19. 13. xi. 23. Hope Nose S by W, The Ness N. Fine red sand.

Station F2I. 13. xi. 23. Black Head SW, Babbacombe NW by W. Very fine reddish sand.

Station F 22. I3. xi. 23. Flat Rock SW $\frac{1}{2} W$, Black Head NW $\frac{1}{4} W$. Coarse muddy shale (unsuitable for grab work).

Station F 39. I6. xi. 23. Clerk Point W by N, Straight Pt. N by E. Coarse muddy gravel.

\begin{tabular}{|c|c|c|c|c|c|c|}
\hline $\begin{array}{l}\text { Station } \\
\text { Sounding (m.) }\end{array}$ & $\begin{array}{l}\mathrm{F}_{15} \\
\text { I5 }\end{array}$ & $\begin{array}{c}\mathrm{F}_{16} \\
\text { I0 }\end{array}$ & $\begin{array}{l}\text { Fig } \\
\text { I0 }\end{array}$ & $\begin{array}{l}\mathrm{F}_{21} \mathrm{I} \\
\mathrm{I} 4\end{array}$ & $\begin{array}{c}\mathrm{F}_{22} \\
22\end{array}$ & $\begin{array}{l}\mathrm{F}_{39} \\
22\end{array}$ \\
\hline \multirow{2}{*}{\multicolumn{7}{|c|}{$\begin{array}{l}\text { CoElEnterata } \\
\text { Hydroid }\end{array}$}} \\
\hline & .. & .. & .. & .. & .. & + \\
\hline \multicolumn{7}{|l|}{ Polychaeta } \\
\hline Aphroditidae & $\cdots$ & . & .. & $\cdots$ & . & I \\
\hline ?Sigalion $\mathrm{sp}$. & .. & I & .. & .. & $\cdots$ & .. \\
\hline Sthenelais boa & . & .. & .. & .. & $\cdots$ & I \\
\hline Phyllodoce maculata & . & .. & .. & .. & .. & I \\
\hline Nereis sp. & $\ddot{7}$ & $\ddot{x}$ & $\ddot{6}$ & $\cdots$ & I & \\
\hline Nephthys sp. & 7 & I & 6 & Io & I & I \\
\hline Glycera sp. & .. & .. & .. & $\cdots$ & I & 6 \\
\hline Magelona sp. & . & .. & .. & .. & . & I \\
\hline Scalibregma sp. & . & .. & .. & . & 2 & 8 \\
\hline Ammotrypane sp. & I & . & .. & $\cdots$ & . & .. \\
\hline Notomastus sp. & .. & .. & . & + & + & .. \\
\hline Owenia fusiformis & 5 & $\because$ & I6 & I & 5 & .. \\
\hline Amphicteis sp. & .. & I & .. & .. & .. & $\because$ \\
\hline Melinna palmata & ++ & .. & .. & c. 9 & 2 & + \\
\hline Amphitrite sp. & $\cdots$ & . & . & & .. & I \\
\hline Lanice conchilega & c. IO & .. & I6 & 48 & .. & . \\
\hline Terebellid & $\because$ & $\because$ & .. & I & .. & .. \\
\hline \multirow{2}{*}{\multicolumn{7}{|c|}{ GEPHYREA }} \\
\hline & & & & & & \\
\hline $\begin{array}{l}\text { Phascolosoma } \mathrm{sp} \text {. } \\
\text { Phascolion strombi }\end{array}$ & $\cdots$ & . & .. & .. & 4 & $\because$ \\
\hline $\begin{array}{l}\text { Phascolion strombi } \\
\text { CRUSTACEA }\end{array}$ & $\cdots$ & $\cdots$ & . & $\cdots$ & $\cdots$ & 2 \\
\hline \multicolumn{7}{|l|}{ CRUSTACEA } \\
\hline $\begin{array}{l}\text { Ampelisca sp. } \\
\text { Bathyporeia sp. }\end{array}$ & .. & $\because$ & $\ddot{2}$ & 8 & $\cdots$ & 0 \\
\hline $\begin{array}{l}\text { Bathyporeia sp. } \\
\text { ?Hippomedon sp. }\end{array}$ & I & $\therefore$ & $\ldots$ & $\because$. & $\cdots$ & $\cdots$ \\
\hline $\begin{array}{l}\text { ?Hippomedon sp. } \\
\text { Eupagurus sp. }\end{array}$ & $\ldots$ & . & .. & $\because$ & :. & $\ddot{I}$ \\
\hline Nika edulis & . & .. & .. & I & $\because$. & 1 \\
\hline Portunus sp. & .. & .. & .. & .. & $\therefore$ & $\ddot{I j}$ \\
\hline \multicolumn{7}{|l|}{ Mollusca } \\
\hline Nucula nitida & 4 & I & .. & 7 & I & .. \\
\hline Thyasira flexuosa & 6 & .. & .. & I & I & I \\
\hline Diplodonta rotundata & . & .. & .. & . & . & I \\
\hline Mysella bidentata & 2 & $\because$ & .. & $\because$ & . & .. \\
\hline Tellina fabula & $\because$. & I & .. & I & .. & .. \\
\hline Abra alba & $3 \mathrm{j}$. & $2 \mathrm{j}$. & .. & $7 \mathrm{j}$. & $2 j$. & .. \\
\hline Abra nitida & 2 & .. & . & $\cdots$ & $\cdots$ & .. \\
\hline Donax vittatus & $\cdots$ & $\because$ & I & . & . & .. \\
\hline Spisula sp. & . & I j. & .. & .. & .. & .. \\
\hline Lutraria lutraria (siphons) & $\because$ & 4 & .. & . & .. & .. \\
\hline Chione striatula & I & $\because$ & . & . & .. & .. \\
\hline Aloidis gibba & $\cdots$ & I 1. & . & . & .. & .. \\
\hline Ensis sp. & . & I j. & .. & .. & .. & .. \\
\hline Cultellus pellucidus & 3 & .. & .. & 2 & .. & .. \\
\hline Turritella communis & .. & . & .. & .. & .. & I \\
\hline \multicolumn{7}{|l|}{ ECHINODERMATA } \\
\hline Amphiura filiformis & 4 & $\cdots$ & $\cdots$ & I & .. & 3 \\
\hline Psammechinus miliaris & $\cdots$ & . & . & $\cdots$ & .. & I \\
\hline Echinocardium cordatum & . & I & .. & .. & .. & . \\
\hline Cucumaria elongata & . & . & . & . & . & 2 \\
\hline Synapta sp. & I & .. & .. & $\cdots$ & .. & \\
\hline
\end{tabular}

For abbreviations, see Table I. 
Table X. SAmples taken by Mr Ford in the 'Corner' Region. Two Hauls of $\frac{1}{10} M .^{2}$ Petersen Grab at Each Station

Note

Station F58. 20. xi. 23. Berry Head N 5 miles. Fine sandy mud.

Station F 59. 20. xi. 23. Berry Head N ro miles. Silty sand, gravel, and shells.

Station F60. 20. xi. 23. Start Point W by N $\frac{3}{4}$, Downend Beacon N by $\mathrm{E}_{4}^{3} \mathrm{E}$. Silty sand, gravel, and shells.

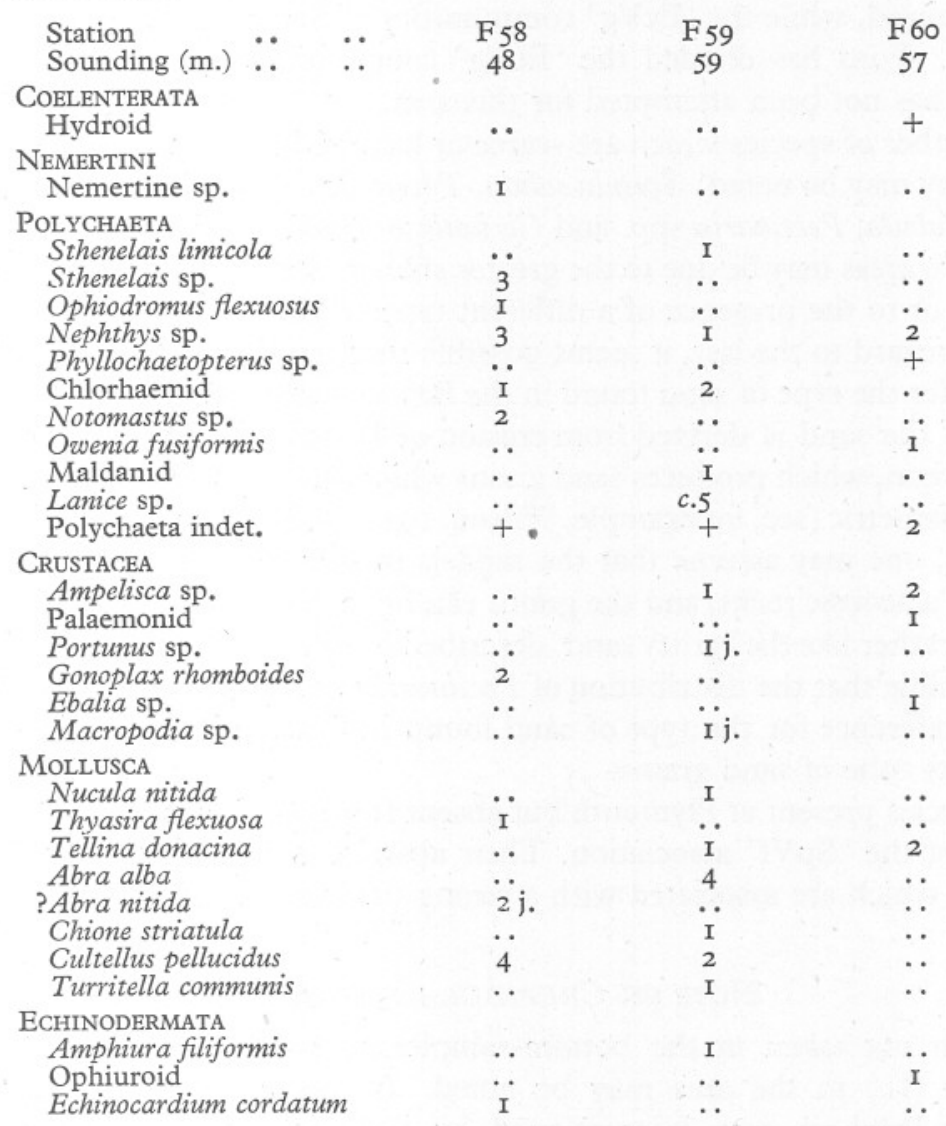

For abbreviations, see Table I.

\section{Comparison with the Plymouth Fauna}

Ford (1923) has outlined the communities to be found on the level sea bottom at Plymouth; he found that the main communities represented were Venus communities with Spatangidae. These were subdivided into two series: Series A, found in finer deposits and characterized by Chione (Venus) striatula and Echinocardium cordatum; and Series B, found in coarse deposits and characterized by Chione (Venus) fasciata and Spatangus purpureus. 
Of forty species listed in Series A, thirty are recorded from Great West Bay; whereas of eighteen in Series B, only two have been taken (Nucula radiata and Chione fasciata), each occurring at only one station. (Glycimeris glycimeris and Laevicardium crassum, both from Series B, have, however, been taken in dredgings about 3 miles south of Straight Point.)

In general, the 'SpVf' communities of Series B are poorly represented and very localized, while the ' $\mathrm{EcVg}$ ' communities of Series A are characteristic of the area. Ford has divided the ' $\mathrm{EcVg}$ ' communities into subcommunities, but this has not been attempted for this area.

A number of species which are scarce or localized at Plymouth but common in the Bay may be noted: Spisula solida, Turritella communis, Lutraria lutraria, Tellina fabula, Pectinaria spp. and Cerianthus lloydi. Differences in the fauna of the two areas may be due to the greater shelter, deposition of large quantities of mud, or to the presence of a different type of bottom in the Bay.

With regard to the last, it seems possible that certain members of the fauna may prefer the type of sand found in the Bay to that off Plymouth. In the Bay much of the sand is derived from erosion of Permo-triassic red sandstone, of desert origin, which produces sand grains which, although angular, are more or less isodiametric (see, for example, Wilson, 1948, pl. XVI, fig. 3). Off Plymouth, however, one may assume that the sand is mainly derived from the marineeroded Palaeozoic rocks, and the grains can be seen to present a more irregular outline, rather like the 'gritty sand' described by Wilson (I948, pl. XVII, fig. 3). It is possible that the distribution of Pectinaria spp., for example, is controlled by its preference for the type of sand found in Great West Bay, since it constructs its tube of sand grains.

Of species present at Plymouth but absent from the Bay one may merely list species of the 'SpVf' association. Their absence is due to the lack of coarse deposits which are associated with a strong tidal scour.

\section{NOTE ON CREPIDULA FORNICATA}

Although not taken in the bottom-sampler hauls, the spread of Crepidula fornicata (L.) to the area may be noted. Its occurrence in the TorquayPaignton-Brixham area is mentioned by Cole (I950). In August I948 a single specimen was found on a whelk shell taken south of Straight Point, and since then the species seems to have increased in numbers. Although the shores of Dawlish Warren and Exmouth have been frequently visited in the past few years, no shells of Crepidula were found until January I950, when a number of empty whelk shells washed up on Dawlish Warren were found to have living Crepidula attached. Most were quite small, a centimetre or two in length, and several chains of three or four individuals were taken. 


\section{SUMMARY}

An account is given of the bottom-fauna of Great West Bay from samples taken in 1948 with a new sampler. Comparison is made with a similar survey made by $\mathrm{Mr}$ E. Ford with the Petersen grab in 1923.

The deposits contain a fair proportion of mud, and the 'EcVg' communities defined by Ford dominate the area. Within the Bay are found fairly dense beds of Turritella communis, Lutraria sp., Callianassa subterranea and Spisula solida.

No marked changes in the fauna seem to have occurred since the earlier survey. The fauna is briefly compared with that of the Plymouth area.

\section{REFERENCES}

Cole, H. A., 1950. The British oyster industry and its problems. Cons. Int. Explor. Mer, Rapp. Proc. Verb., (in the press).

Ford, E., I923. Animal communities of the level sea bottom in the waters adjacent to Plymouth. Fourn. Mar. Biol. Assoc., Vol xIII, pp. I64-224.

- 1925. On the growth of some lamellibranchs in relation to the food supply of fishes. Fourn. Mar. Biol. Assoc., Vol. xiII, pp. 53I-59.

GARSTANG, W., I903. Report on trawling and other investigations carried out in the bays on the south-east coast of Devon during I90I and 1902. Fourn. Mar. Biol. Assoc., Vol. vI, pp. 435-527.

Holme, N. A., I949. A new bottom sampler. Fourn. Mar. Biol. Assoc., Vol. xxviII, pp. 323-32.

Holt, E. W. L., I898. Report on trawling in bays on the south-east coast of Devon. fourn. Mar. Biol. Assoc., Vol. v, pp. 296-321.

KYLE, H. M., I903. Notes on the physical conditions existing within the line from Start Point to Portland. Fourn. Mar. Biol. Assoc., Vol. vi, pp. 528-40.

Marine Biological Association, 193i. Plymouth Marine Fauna. 2nd. ed.

STEAD, F. B., I896. Preliminary note on trawling experiments in certain bays on the south-east coast of Devon. Fourn. Mar. Biol. Assoc., Vol. Iv, pp. 90-6.

TopD, R. A., I903. Notes on the invertebrate fauna and fish food of the bays between the Start and Exmouth. Fourn. Mar. Biol. Assoc., Vol. vI, pp. 54I-6I.

WILson, D. P., I948. The relation of the substratum to the metamorphosis of Ophelia larvae. fourn. Mar. Biol. Assoc., Vol. xxvII, pp. 723-60. 


\section{APPENDIX}

\section{Table XI. Mr Ford's Stations (1923). S.S. SALPA}

$\frac{-1}{10} \mathrm{~m} \cdot{ }^{2}$ Petersen grab.

F I 4
I5
I6
I7

I8
19
20
21
22
23
31
32
33
34
35
36
37
38
39
58
59
60

I3. xi. 23

,

,

g9

,

,

צפ

פ,

I4. xi. 23

I5. xi. 23

,

,

,

,

,

,

I6. xi. 23

20. xi. 23

,

Hope Nose W $\frac{1}{2}$ S, The Ness NW by W Hope Nose S, Babbacombe Pt. W by S

Babbacombe Pt. S, Petit Tor NW

Orestone $\mathrm{S} \frac{1}{2} \mathrm{~W}, \mathrm{Bab}$ acombe Pt. W by $\mathrm{S}_{2} \mathrm{~S}$

(Berry Head over Flat Rock)

Babbacombe Pt. SW $\frac{1}{2}$ S, The Ness N $\frac{1}{2} \mathrm{E}$

The Ness N, Hope Nose S by W

The Ness NW, Orestone S by W

Black Hd. SW, Babbacombe Pt. NW by W

Flat Rock SW $\frac{1}{2} W$, Black Hd. NW $\frac{1}{4} W$

Orestone NW by W $\frac{1}{2} \mathrm{~W}$, The Ness $\mathrm{N}$ by W $\frac{1}{4} \mathrm{~W}$

Saltern Cove NW, Churstone Pt. SW by W $\frac{1}{2} W$

Red Cliff Hotel NW by N, Roundham Hd. NW by W $\frac{1}{2} W$

Orestone $\mathrm{E}$ by $\mathrm{N}$, Torquay Pier $\mathrm{N}$ by W

Thatcher Rock N, Orestone NE by N

Berry Hd. S, Brixham Breakwater Light SW by W

Brixham Breakwater Light WSW, Berry Hd. SE

Brixham Breakwater Light SE by E, Old Battery SW

Quarries WNW, Fishcombe Pt. $\mathrm{S}_{2}^{1} \mathrm{E}$

Clerk Pt. W by N, Straight Pt. N by E

Berry Hd. N, 5 miles (log)

Berry Hd. N, Io miles (log)

Start Pt. W by $\mathrm{N}_{4}^{3} \mathrm{~N}$, Downend Beacon $\mathrm{N}$ by $\mathrm{E}_{4}^{3} \mathrm{E}$

N.B. Ford's positions are compass bearings, magnetic variation in 1923 being $15^{\prime} \circ 5^{\prime \prime} \mathrm{W}$. 
TABle XII. I948 Stations. R.V. SABELLA

2
3
4
5
6
7
8
9
10
11
12
13
14
15
16
17
18
19
20
21
22
23
24
25
26
27
28
29
30
31
32
33
34
35
36
37

\begin{tabular}{|c|c|c|c|}
\hline \multicolumn{4}{|c|}{$\frac{1}{20} \mathrm{~m} \cdot{ }^{2}$ Scoop-sampler } \\
\hline I & 29. vii. 48 & Exmouth Church & $349^{\circ}, I \cdot 2^{\prime}$ \\
\hline 2 & , & , & $349^{\circ}, \mathrm{I} \cdot 7^{\prime}$ \\
\hline 3 & " & , & $348^{\circ}, 2 \cdot 2^{\prime}$ \\
\hline 4 & " & , & $349^{\circ}, 2 \cdot 7^{\prime}$ \\
\hline 5 & ” & " & $349^{\circ}, 3 \cdot 3^{\prime}$ \\
\hline 6 & ” & " & $349^{\circ}, 3 \cdot 8$ \\
\hline $\begin{array}{l}7 \\
8\end{array}$ & " & $"$ & $349^{\circ}, 4.3^{\prime}$ \\
\hline $\begin{array}{l}8 \\
9\end{array}$ & " & $"$ & $349,4.8$ \\
\hline $\begin{array}{r}9 \\
\text { I0 }\end{array}$ & $"$ & " & $349^{\circ}, 5 \cdot 3^{\prime}$ \\
\hline $\begin{array}{l}\text { IO } \\
\text { II }\end{array}$ & $"$ & $"$ & $\begin{array}{l}349^{\circ}, 5 \cdot 8^{\prime} \\
349^{\circ}, 6 \cdot 2^{\prime}\end{array}$ \\
\hline 12 & 30. vii. 48 & Langstone Point & $\begin{array}{l}349^{\circ}, 0.2 \\
334^{\circ}, 0.35^{\prime}\end{array}$ \\
\hline I3 & , & , & $334^{\circ}, 0.75^{\prime}$ \\
\hline I4 & $"$ & , & $334^{\circ}, 0.55^{\prime}$ \\
\hline I5 & " & " & $022^{\circ}, 0.55^{\prime}$ \\
\hline 16 & " & " & $013^{\circ}, 0.9^{\prime}$ \\
\hline $\begin{array}{l}\text { I7 } \\
\text { I8 }\end{array}$ & " & "' & OI $7^{\circ}, \mathrm{I} \cdot 15^{\prime}$ \\
\hline $\begin{array}{l}18 \\
19\end{array}$ & " & Teignmouth Pier & $229^{\circ}, \mathrm{I} \cdot 7^{\prime}$ \\
\hline $\begin{array}{l}\text { 19 } \\
20\end{array}$ & " & " & $25 \mathrm{I}^{\circ}, \mathrm{I} \cdot 7^{\prime}$ \\
\hline $\begin{array}{l}20 \\
21\end{array}$ & 30. vii. 48 & " & $324^{\circ}, 0 \cdot 6^{\prime}$ \\
\hline 22 & $\begin{array}{l}30 . \\
y, 40\end{array}$ & Orestone & $004^{\circ}, 1 \cdot 9^{\prime}$ \\
\hline 23 & ??.. & , & $\begin{array}{l}210,2.2 \\
222^{\circ}, 3.5^{\prime}\end{array}$ \\
\hline 24 & 4. viii. 48 & Berry Head & $225^{\circ}, 6 \cdot 2^{\prime}$ \\
\hline $\begin{array}{l}25 \\
26\end{array}$ & $"$ & ", & $254^{\circ}, 5 \cdot 7^{\prime}$ \\
\hline 27 & 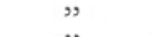 & " & $280^{\circ}, 5^{\circ} 8^{\prime}$ \\
\hline 28 & " & " & $297^{\circ}, 6 \cdot 4^{\prime}$ \\
\hline 29 & ” & $"$ & $310^{\circ}, 8 \cdot 2^{\prime}$ \\
\hline 30 & " & Start Point & $\begin{array}{l}319^{\circ}, 9.4 \\
275^{\circ}, 9^{\prime} 6^{\prime}\end{array}$ \\
\hline $\begin{array}{l}31 \\
32\end{array}$ & " & , & $282^{\circ}, 6 \cdot 3^{\prime}$ \\
\hline $\begin{array}{l}32 \\
33\end{array}$ & " & " & $270^{\circ}, 5 \cdot 4^{\prime}$ \\
\hline $\begin{array}{l}33 \\
34\end{array}$ & $"$ & " & $240^{\circ}, 5^{\cdot 6^{\prime}}$ \\
\hline $\begin{array}{l}34 \\
35\end{array}$ & $"$ & " & $188^{\circ}, 3 \cdot 2^{\prime}$ \\
\hline 36 & $"$ & 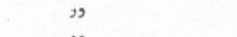 & $181^{\circ}, 3 \cdot 3^{\prime}$ \\
\hline 37 & " - - - - & " & $\begin{array}{l}\text { I81, } 3.5 \\
182^{\circ}, 37^{\prime}\end{array}$ \\
\hline & & ge hauls & \\
\hline II & 3I. vii. 48 & Hope Nose & $\mathrm{I} 77^{\circ}, \mathrm{I} \cdot 6^{\prime}$ \\
\hline III & 3I. vii. $4^{8}$ & Teignmouth Pier & $319^{\circ}, \mathrm{I} \cdot 4^{\prime}$ \\
\hline XVI & 3. viii. 48 & Langstone Point & $298^{\circ}, I \cdot I 2^{\prime}$ \\
\hline
\end{tabular}

N.B. The above are true bearings, and distances are in nautical miles. 